International Journal of Pure and Applied Mathematics

Volume 88 No. $2 \quad 2013,183-211$

ISSN: 1311-8080 (printed version); ISSN: 1314-3395 (on-line version)

url: http://www.ijpam.eu

doi: http://dx.doi.org/10.12732/ijpam.v88i2.4

ijpam.eu

\title{
PARTITIONS-REQUIREMENTS-MATRICES AS OPTIMAL MARKOV KERNELS OF SPECIAL STOCHASTIC DYNAMIC DISTANCE OPTIMAL PARTITIONING PROBLEMS
}

\author{
R. Hildenbrandt \\ Department of Mathematics \\ Ilmenau Technical University \\ PF 1006 65, 98684 Ilmenau, GERMANY
}

\begin{abstract}
The Stochastic Dynamic Distance Optimal Partitioning problem (SDDP problem) is a complex Operations Research problem. The SDDP problem is based on an industrial problem, which contains an optimal conversion of machines.

Partitions of integers as states of these stochastic dynamic programming problems involves combinatorial aspects of SDDP problems. Under the assumption of identical "basic costs" (in other words of "unit distances") and independent and identically distributed requirements we will show (in many cases) by means of combinatorial ideas that decisions for feasible states with least square sums of their parts are optimal solutions. Corresponding Markov kernels are called partitions-Requirements-Matrices (PRMs).

Optimal decisions of such problems can be used as approximate solutions of corresponding SDDP problems, in which the basic costs differ only slightly from each other or as starting decisions if corresponding SDDP problems are solved by iterative methods, such as the Howard algorithm.
\end{abstract}

AMS Subject Classification: 90C40, 05A99, 15B51, 15B36, 90B30, 90B06 Key Words: Markov decision process, combinatorics, stochastic dynamic distance optimal partitioning problem, partitions-requirements-matrices, production, transport

Received: June 25, 2013

(c) 2013 Academic Publications, Ltd. url: www.acadpubl.eu 


\section{Introduction}

The Stochastic Dynamic Distance Optimal Partitioning (SDDP) problem is a complex Operations Research problem. The SDDP problem is based on a problem in industry, which contains an optimal conversion of machines. Partitions of integers as states of these stochastic dynamic programming problems involves combinatorial aspects of SDDP problems, which can also be observed in matrices of transition probabilities (Markov kernels) and average one-step reward functions of SDDP problems, modelled as Markov decision processes (MDPs). SDDP problems modelled as MDPs can be exactly solved by means of the (modified) Howard algorithm. However, if the number of states is very large, then the computation of exact solutions is hardly realizable. Investigations of special cases or of inherent characteristic structures of SDDP problems are also important as a basis for heuristics.

Under the assumption of identical "basic costs" (in other words of "unit distances") and independent and identically distributed requirements decisions for feasible states with least square sums of their parts are optimal solutions, as is shown (in many cases) toward the end of this paper. However, we will also see that is not simple to solve even such special cases of SDDP problems or to prove that optimally conjectured decisions of these problems are in fact optimal. Under the special assumptions we will use an optimality criterion (monotone solutions of Poisson equations) which was be derived from the Howard algorithm. Thereby the corresponding matrices of transition probabilities which are called Partitions-requirements-matrices (PRMs) come into focus. The definition of PRMs includes that PRMs can be initially computed by means of simple enumeration, however a laborious method. In addition, there is a main difficulty to deal with: No formulas are known for most of the elements in PRMs. Thus, PRMs themselves represent interesting (almost self-evident) combinatorial structures, which are not otherwise found in literature. Instead of formulas we will use limits of elements of certain PRMs, which will be computed in Section 6. For this purpose, we will define sets of "sparse partitions" and sets of "non-truncated heavy partitions".

The monotonicity of solutions of Poisson equations will be proven in many cases. The solutions of the Poisson equations, with regard to the limits of PRMs, have an elegant structure, in contrast to the formulas for the limits of PRMs. These solutions include in relation to the distribution of requirements generalized harmonic numbers.

Optimal decisions of such special SDDP problems can be used as approximate solutions of corresponding SDDP problems, in which the basic costs differ 
only slightly from each other or as starting decisions if corresponding SDDP problems are solved by iterative methods, such as the Howard algorithm, for example.

In this paper we focus on combinatorial ideas. We will refer to corresponding literature for statements which are based on the theory of MDPs. Furthermore, let $\mathbb{Z}$ denote the set of integers, $\mathbb{N}$ the set of positive integers and $\mathbb{Z}_{+}^{n}$ the set of $\mathrm{n}$-dimensional integer vectors with nonnegative coordinates.

\section{Introduction to SDDP Problems}

Parts of different types are produced by means of machines. For this purpose, the machines have to be converted into states, which are in accordance with the types of the parts. ${ }^{1}$ Thereby, costs are incurred. Each machine can be converted into each state.

The number of machines is denoted by $s u \in \mathbb{N}$ and the number of types of parts by $n \in \mathbb{N}, n \geq 2$. Let $\tilde{s}_{i}$ denote the number of machines in state $i$ and $\tilde{s}=\left(\tilde{s}_{1}, \tilde{s}_{2}, \cdots, \tilde{s}_{n}\right)$. Then $\sum_{i=1}^{n} \tilde{s}_{i}=s u$ follows. (The su machines in the different states correspond to a partition of $s u$ ). If a machine is converted from a state $i(i \in\{1,2, \cdots, n\})$ into a state $j(j \in\{1,2, \cdots, n\})$ (which are in accordance with the types $i$ and $j$ of the parts), then the incurred (basic) cost are denoted by $k_{i j} \in \mathbb{R}_{+}$.

The production takes place in successive (equidistant) stages (or periods). In one stage one part can be produced (at most) by one machine. In each stage a requirement of parts (of several types) is to be met. At first, probability functions (denoted by $q_{i}(i \in\{1,2, \cdots, n\})$ ) of the requirements are given. The realizations of the requirements for a stage are known at the beginning of the stages (before the decision of conversions of machines has to be made). The maximum number of produced parts in a stage of type $i \quad(i \in\{1,2, \ldots, n\})$ is denoted by $k_{0_{i}}$. The stages are numbered by subscript $t, t=1,2, \cdots$. We use the same notations $w_{t}$, for the random requirements of parts of different types and their realizations $\left(w_{t} \in \mathbb{Z}_{+}^{n}\right.$ and $\left.0 \leq w_{t, i} \leq k_{0_{i}}\right)$ at stage $t . B_{n ; k_{0}}:=$ $\left\{w \in \mathbb{Z}_{+}^{n} \mid 0 \leq w_{t, i} \leq k_{0_{i}}\right\}$ is called the set of requirements. Principally, two cases in relation to the requirements have to be considered at each stage:

$$
-C\left[w_{t}, s u\right]: \text { denotes the case } \sum_{i=1}^{n} w_{t, i} \leq s u,
$$

\footnotetext{
${ }^{1}$ One may think, for example, of concrete moulds, see [4], Section 1.2.
} 
which means the requirements can be completely fulfilled.

$$
-C\left[s u, w_{t}\right]: \text { denotes the case } \sum_{i=1}^{n} w_{t, i} \geq s u^{2}
$$

in which the requirement $w_{t}$ cannot be completely met, if " $>$ " is valid. In this case the machines $s u$ are not sufficient. However, in no case should availabilities be dissipated. ${ }^{3}$

The objective is to minimize the average expected cost per stage in the case of an infinite horizon. Therefore it must be decided which machine is to be converted to which state in each stage.

In other words, at every stage $t(t=1,2, \ldots)$ with a current $\tilde{s}_{t}$ and an observed $w_{t}$ at the beginning of the stage such $\tilde{s}_{t+1}$ with

$$
\left\{\begin{array}{l}
w_{t} \leq \tilde{s}_{t+1} \text { in } C[w, s u] \\
\tilde{s}_{t+1} \leq w_{t} \text { in } C[s u, w]
\end{array}\right.
$$

are to be found so that altogether the average expected cost per stage will be minimal. Thus, SDDP problems are stochastic dynamic programming problems with random disturbances. 4 The state space is set of the ordered restricted partitions with at most $n$ parts, whereby the elements are written as vectors:

$$
\tilde{S}_{n ; s u ; k_{0}}=\left\{\tilde{s} \in \mathbb{Z}_{+}^{n} \mid 0 \leq \tilde{s}_{i} \leq k_{0_{i}}, \sum_{i=1}^{n} \tilde{s}_{i}=s u\right\} .
$$

${ }^{5}$ We will consider stationary models. This means $k_{i j}, k_{0_{i}}$ and the probability functions $q_{i}$ of the requirements $(i=1, \ldots, n, j=1, \ldots, n)$ are the same in all stages. Hence in most cases, we do not need the subscript $t$. Instead, we use the inferior indices $n, k_{0}$ and $s u$ as characteristics of the concrete SDDP problems. The formulation of distance properties for stochastic dynamic programming

\footnotetext{
${ }^{2}$ In this instance it is not necessary to distinguish the case $\sum_{i=1}^{n} w_{t, i}=s u$.

${ }^{3}$ If a possibility for the storage of parts would be given, then the mathematical model could be extended as follows. In periods where case C[w, su] (with a "<"-sign) is present, additional parts could be produced as reserve for periods where the production capacity is not sufficient. However, the extension of the mathematical model would be determined by the concrete aims of the production process and the detailed storage possibilities.

${ }^{4}$ For dynamic models, where the requirements are deterministic, see [10].

${ }^{5}$ The additional symbol " $~ "$ is attached to $s$ in order later to differentiate ordered from unordered partitions. Unordered partitions will be states of certain reduced SDDP problems (compare Section 3).
} 
problems led to the fact that the machines are not converted if they are not needed to produce parts for the requirements of a stage $t .{ }^{6}$ Then the minimum will not increase, when following smaller sets $\hat{A}_{n ; s u ; k_{0}}\left(\tilde{s}_{t}, w_{t}\right)$ are used instead of (3) (see [6], Theorem 6).

$$
\begin{gathered}
\left\{\begin{array}{l}
\hat{A}_{n ; s u ; k_{0}}(\tilde{s}, w):= \\
\tilde{s}^{\prime} \in \tilde{S}_{n ; s u ; k_{0}} \mid \begin{array}{l}
w_{i} \leq \tilde{s}_{i}^{\prime} \leq \max \left\{\tilde{s}_{i}, w_{i}\right\}, i=1, \cdots, n, \text { in } C[w, s u] \\
\min \left\{\tilde{s}_{i}, w_{i}\right\} \leq \tilde{s}_{i}^{\prime} \leq w_{i}, i=1, \cdots, n, \text { in } C[s u, w]
\end{array} \\
\left(\tilde{s} \in S_{n ; s u ; k_{0}}, w \in B_{n ; k_{0}}\right)
\end{array}\right\}
\end{gathered}
$$

is called the decision set for the SDDP problem.

In the following we will not consider the general model of the SDDP problem any more. We suppose the following special properties.

\section{The Conjecture about Optimal Decisions for SDDP Problems with Identical Basic Costs and Independent and Identically Distributed Requirements}

Throughout the further sections we suppose

$$
k_{i j}=1 \forall i \neq j \text { and } k_{i i}=0 \forall i .
$$

Let $\hat{c}\left(\tilde{s}, \tilde{s}^{\prime}\right)$ (or $\left.\hat{c}(\tilde{s}, w)\right)$ denote the minimal cost of the (non-balanced) classical transportation problems with availabilities $\tilde{s}$, requirements $\tilde{s}^{\prime}$ (or $w$ ) and unit distances. If $\tilde{s}=\tilde{s}_{t} \in \tilde{S}_{n ; s u ; k_{0}}, \tilde{s}^{\prime}=\tilde{s}_{t+1} \in \hat{A}_{n ; s u ; k_{0}}(\tilde{s}, w)$ and $w=w_{t} \in$ $B_{n ; k_{0}}$ then $\hat{c}\left(\tilde{s}, \tilde{s}^{\prime}\right)$ include the sum of all conversion costs at stage $t$ and the relationships

$$
\begin{aligned}
\hat{c}\left(\tilde{s}, \tilde{s}^{\prime}\right)=\hat{c}(\tilde{s}, w)= & \left\{\begin{array}{l}
\sum_{i} \max \left\{0, w_{i}-\tilde{s}_{i}\right\} \quad \text { in case } C[w, s u] \\
\sum_{i} \max \left\{0, \tilde{s}_{i}-w_{i}\right\} \quad \text { in case } C[s u, w]
\end{array}\right. \\
& \forall \tilde{s}^{\prime} \in \hat{A}_{n \cdot s u ; k_{0}}(\tilde{s}, w)
\end{aligned}
$$

can be simply shown. In case of independent and identically distributed requirements $k_{0_{1}}=k_{0_{2}}=\cdots=k_{0_{n}}=: k_{0}$ and

$$
k_{0} \leq s u<n k_{0}
$$

\footnotetext{
${ }^{6}$ Corresponding algorithms with this property are called "lazy algorithms", see Section 10.2.3 in [1].
} 
are assumed and

$$
q(w):=\prod_{i=1}^{n} q_{0}\left(w_{i}\right)
$$

denote the probability for $w \in B_{n ; k_{0}}$, where the marginal or single probabilities $q_{0}\left(w_{i}\right)$ are such that

$$
q_{0}\left(w_{i}\right)>0 \text { for } w_{i} \in\left\{0,1, \cdots, k_{0}\right\} \text { and } \sum_{j=0}^{k_{0}} q_{0}(j)=1 .
$$

The average one-step reward functions are computed as follows

$$
\gamma(\tilde{s})=\sum_{w: w \in B_{n ; k_{0}}} \hat{c}(\tilde{s}, w) q(w) \quad\left(\text { for } \tilde{s} \in \tilde{S}_{n ; s u ; k_{0}}\right)
$$

(According to (7), they are the same for all $\tilde{s}^{\prime} \in \hat{A}_{n ; s u ; k_{0}}(\tilde{s}, w)$ in our special case.)

Theorem 1. $\quad \gamma(\tilde{s})=\sum_{i=1}^{n} \sum_{w_{i}=0}^{\tilde{s}_{i}}\left(\tilde{s}_{i}-w_{i}\right) q_{0}\left(w_{i}\right)+R\left(n, s u, k_{0}, q\right)$, where $R\left(n, s u, k_{0}, q\right)=\sum_{w \in B_{n} ; k_{0}: C[w, s u]} q(w)\left(\sum_{i=1}^{n} w_{i}-s u\right)$ is independent of $\tilde{s}$.

(For the proof see [4], Lemma 3.12.)

Under the assumptions of identical basic costs and independent and identically distributed requirements, the corresponding SDDP problems can be reduced such that we can use the set $S_{n ; s u ; k_{0}}$ of the unordered partitions of $s u$ with at most $n$ parts with summands not greater than $k_{0}$, (where the elements of $S_{n ; s u ; k_{0}}$ are expressed as $n$-dimensional vectors, meaning $s=\left(s_{1}, s_{2}, \cdots, s_{n}\right)$ ) as the reduced state space corresponding to $\tilde{S}_{n ; s u ; k_{0}}$ (see [4], Section 3.3). (Partitions $s$ and $s^{\prime}$ are equal if $s^{\prime}$ is a permutation of $s$.) Furthermore, let $r:=\left|S_{n ; s u ; k_{0}}\right|$ be the number of the restricted partitions.

$$
\begin{aligned}
& \hat{A}_{n ; s u ; k_{0}}(s, w) \\
& =\left\{\begin{array}{l|l}
s^{\prime} \in S_{n ; s u ; k_{0}} & \begin{array}{l}
\exists s_{\pi}^{\prime} \text { permutation of } s^{\prime}: \\
w_{i} \leq s_{\pi i}^{\prime} \leq \max \left\{s_{i}, w_{i}\right\}, i=1, \cdots, n, \text { in } C[w, s u], \\
\min \left\{s_{i}, w_{i}\right\} \leq s_{\pi i}^{\prime} \leq w_{i}, i=1, \cdots, n, \text { in } C[s u, w]
\end{array}
\end{array}\right\} .
\end{aligned}
$$

are then the decision sets for the reduced SDDP problem.

$s^{\prime}=s^{\prime}(s, w) \in \hat{A}_{n ; s u ; k_{0}}(s, w)$ are called feasible states or partitions with respect to $s$ and $w$. 
We can now make the following conjecture:

Decisions for feasible states $s$ with minimum $\gamma(s)$ are optimal if (6), (9) and (10) are supposed.

Furthermore, we will see that conjecture (Con) is equivalent to:

Decisions for feasible states with least square sums of their components are optimal if (6) (9) and (10) are supposed.

(See Lemma 2 in the following section.)

We will see that is not simple to prove that optimally conjectured decisions of these problems are in fact optimal. From that point on, we will deal in detail with conjecture (Con') and will prove this conjecture for many cases by means of combinatorial ideas.

\section{Partitions-Requirements-Matrices, Poisson Equations and an Optimality Criterion}

For $y \in \mathbb{Z}_{+}^{n}$ we define the vector $y\left[i_{1} ; i_{2}\right]$ with

$$
y_{i}\left[i_{1} ; i_{2}\right]:=\left\{\begin{array}{lll}
y_{i}+1 & \text { for } & i=i_{1}, \\
y_{i}-1 & \text { for } & i=i_{2}, \\
y_{i} & \text { otherwise } &
\end{array}\right.
$$

If it is necessary, we use the (partial) dominance or majorization ordering on unordered restricted partitions which implies that

- $s^{f} \in S_{n ; s u ; k_{0}}$ is a direct predecessor of $s^{l} \in S_{n ; s u ; k_{0}}$ if and only if $\exists i_{1}, i_{2}:\left(s_{i_{1}}^{l} \geq s_{i_{2}}^{l}\right) \wedge\left(s^{l}=s^{f}\left[i_{2} ; i_{1}\right]\right)\left(\right.$ where $s_{1}^{y} \geq s_{2}^{y} \geq \cdots \geq s_{n}^{y}$ for $y=1,2)$.

This is equivalent to

- permutations $s_{\pi}^{f}$ of $s^{f}$ and $s_{\pi}^{l}$ of $s^{l}$ exist such that

$\frac{1}{2} \sum_{i}\left|s_{\pi_{i}}^{f}-s_{\pi_{i}}^{l}\right|=1$ and $\sum_{i}\left(s_{i}^{f}\right)^{2}>\sum_{i}\left(s_{i}^{l}\right)^{2}$.

(See [7], Marshall, A.W. and Olkin, I.,Chapter 1. A., B. and Chapter 5. D. and see also [3].)

A feasible partition $s^{*}=s^{*}(s, w) \in \hat{A}_{n ; s u ; k_{0}}(s, w)$ with respect to $s$ and $w$ is called the feasible balanced partition (or state) with respect to $s$ and $w$ if $s^{*}$ is an optimal solution of the problem 


$$
\sum_{i=1}^{n}\left(s_{i}^{*}\right)^{2} \rightarrow \min \quad \text { subject to } s^{*} \in \hat{A}_{n ; s u ; k_{0}}(s, w) \text { and }
$$

$s^{*}=s^{*}(s, w)$ is also called a feasible balanced transition from $s$ to $s^{*}$ for $w$. In addition, $B_{n, k_{0}}^{*}\left(s, s^{*}\right)=\left\{w \in B_{n, k_{0}} \mid s^{*}=s^{*}(s, w)\right\}$ is the set of balancing requirements.

In the following Lemma connections with the average one-step reward functions and the decision for feasible states with the least square sums of their components are given.

Lemma 2. Let $S_{n ; s u ; k_{0}}=\left\{s^{1}, s^{2}, \cdots, s^{r}\right\}$ and $B_{n ; k_{0}}$ be given and let the requirements $w_{i},(i=1, \cdots, n)$ be independent and identically distributed, where (10) is additionally assumed.

(a) If $s^{\prime} \in \hat{A}_{n ; s u ; k_{0}}(s, w)$, then $s^{*}=s^{*}(s, w)$ is a successor of $s^{\prime}$.

(b) If $s^{l} \in S_{n ; s u ; k_{0}}$ is a successor of $s^{f} \in S_{n ; s u ; k_{0}}\left(s^{f} \neq s^{l}\right)$, then $\gamma\left(s^{f}\right)>\gamma\left(s^{l}\right) \Leftrightarrow \sum_{i=1}^{n}\left(s_{i}^{f}\right)^{2}>\sum_{i=1}^{n}\left(s_{i}^{l}\right)^{2}$.

((a): for the proof see [4], Theorem 3.22. (b): Intially, we can show the conjecture for a direct successor $s^{l}$ of $s^{f}$ (using Theorem 1) from which (b) then follows.)

Definition 3. Let $S_{n ; s u ; k_{0}}=\left\{s^{1}, s^{2}, \cdots, s^{r}\right\}$ and $B_{n ; k_{0}}$ be given and let the requirements $w_{i},(i=1, \cdots, n)$ be independent and identically distributed, where $(10)$ is additionally assumed. $P^{*}=P_{n ; s u ; k_{0}}^{*}=\left(p_{f l}^{*}\right)$ with elements

$$
p_{f l}^{*}=p^{*}\left(s^{l} \mid s^{f}\right)=\sum_{w \in B_{n, k_{0}}^{*}\left(s^{f}, s^{l}\right)} q(w) \text { for } f=1, \cdots, r, l=1, \cdots, r
$$

is called the partitions-requirements-matrix (PRM).

(In other words, PRMs are matrices of transition probabilities for reduced SDDP problems in case of feasible balanced transition.) Obviously, since $p_{f l}^{*}>$ $q\left(w=s^{l}\right)$,

$$
p_{f l}^{*}>0
$$

An optimality criterion for reduced SDDP problems, which based on Poisson equations, can be derived from the Howard algorithm (policy iteration) for MDPs.

Definition 4. Let a set of requirements $B_{n ; k_{0}}$ and a set $S_{n ; s u ; k_{0}}=$ $\left\{s^{1}, s^{2}, \cdots, s^{r}\right\}$ be given and let the numbering of the partitions of the set 
of restricted partitions be such that if $s^{f}$ is a (direct) predecessor of $s^{l}$ then $f<l$. Let the requirements $w_{i},(i=1, \cdots, n)$ be independent and identically distributed, where (10) is additionally assumed. Furthermore, let $P^{*}=$ $\left(p_{f l}^{*}\right)$ be the the corresponding PRM. The vector equations (with the variables $\left.(g, \nu) \in \mathbb{R} \times \mathbb{R}^{r}\right)$

$$
g(-1, \cdots,-1)^{T}+\left(P^{*}-I\right) \nu=-\gamma^{\prime}
$$

are called the Poisson equations for the PRM $P^{*}$, where I is the identity matrix,

$$
\gamma_{f}\left(=\gamma\left(s^{f}\right)\right)=\sum_{i=1}^{n} \sum_{w_{i}=0}^{s_{i}^{f}}\left(s_{i}^{f}-w_{i}\right) q_{0}\left(w_{i}\right) \text { for } f=1,2, \cdots, r
$$

and $\gamma^{\prime}$ any affine transformation of $\gamma$ :

$$
\gamma^{\prime}=\alpha \gamma+\beta(1, \cdots, 1)^{T} \text { with } \alpha>0 .
$$

It is well-known that if one variable $\nu_{f_{0}}$ is fixed in any way, then under the condition (14), for example, the remaining equation system has an unique solution. (See the proof of Theorem 2.4.8 by Müller and Nollau [8], for instance).

Definition 5. A solution of a Poisson equation for a PRM, as in Definition 4 , is called monotone (in $\nu$ ) (with respect to the partial order) if $s^{f}$ is a (direct) predecessor of $s^{l}$ then $\nu_{f}>\nu_{l}$.

We can now make the following conjecture:

Solutions of all Poissons equation for PRMs are monotone.

(Con")

A specification of the Howard algorithm for $\operatorname{MDPs}^{7}$ (with average reward criterion) leads to the following optimality criterion for reduced SDDP problems in case of feasible balanced transition.

Optimality criterion: If a solution of a Poisson equation for a PRM is monotone, then decisions for feasible states (unordered partitions) with the least square sums of their components are optimal for the corresponding reduced SDDP problems.

\section{Computations of PRMs by Means of an Iterative Method}

Feasible balanced partitions for given $s \in S_{n ; s u ; k_{0}}$ and $w \in B_{n, k_{0}}$ can be computed by the following iterative method ([4], Lemma 3.13):

\footnotetext{
${ }^{7}$ A detailed description of the Howard algorithm can be found, for example, in [8], Section 2.4.2.1 or in [9], Section 8.6.
} 
Set $s^{*^{\prime}}=s$.

(*) If (11) is satisfied, then $s^{*^{\prime}}$ is the desired partition (end) else:

Determine a component $s_{j}^{*^{\prime}}$ of $s^{*^{\prime}}$ with

$$
s_{j}^{*^{\prime}}=\left\{\begin{array}{l}
\max \left\{s_{i}^{*^{\prime}} \mid s_{i}^{*^{\prime}}>w_{i}\right\} \text { in } C[w, s u], \\
\min \left\{s_{i}^{*^{\prime}} \mid s_{i}^{*^{\prime}}<w_{i}\right\} \text { in } C[s u, w]
\end{array}\right.
$$

and a component $s_{y}^{*^{\prime}}$ with

$$
\left\{\begin{array}{l}
s_{y}^{*^{\prime}}<w_{y} \text { in } C[w, s u] \\
s_{y}^{*^{\prime}}>w_{y} \text { in } C[s u, w]
\end{array} .\right.
$$

Set

$$
s_{j}^{*^{\prime}}=\left\{\begin{array}{l}
s_{j}^{*^{\prime}}-1 \text { in } C[s u, w] \\
s_{j}^{*^{\prime}}+1 \text { in } C[s u, w]
\end{array}\right.
$$

and

$$
s_{y}^{*^{\prime}}=\left\{\begin{array}{l}
s_{y}^{*^{\prime}}+1 \text { in } C[s u, w] \\
s_{y}^{*^{\prime}}-1 \text { in } C[s u, w]
\end{array} .\right.
$$

Go to $\left({ }^{*}\right)$.

Then, the elements of PRMs $p_{f l}^{*}$ can be computed as sum of the probabilities of the requirements $w$, for which feasible balanced transitions from $s^{f}$ to $s^{l}$ are given (see Definition 3).

Example 5.1. Let $n=3, k_{0}=3, s u=6$ and $q_{0}\left(w_{i}\right)=\frac{1}{1+k_{0}}$ $=\frac{1}{4}$ for $w_{i}=0,1,2,3$ be given (hence $q(w)=\left(\frac{1}{4}\right)^{3}=\frac{1}{64}$ for all $\left.w \in B_{3 ; 3}\right)$. Then the set $S_{3 ; 6 ; 3}$ includes the elements $s^{1}=(3,3,0)^{T}$, $s^{2}=(3,2,1)^{T}, s^{3}=(2,2,2)^{T}$. Feasible balanced partitions are, for instance, $s^{*}\left(s^{1}=(3,3,0)^{T}, w=(0,0,2)^{T}\right)=(2,2,2)^{T}\left(=s^{3}\right)$,

$$
s^{*}\left(s^{1}=(3,3,0)^{T}, w=(3,2,3)^{T}\right)=(3,2,1)\left(=s^{2}\right) .
$$

However, in order to compute the PRM we need to know all of the $r \cdot\left(k_{0}+1\right)^{n}$ feasible balanced partitions (with respect to all $\left.s \in S_{n ; s u ; k_{0}}, w \in B_{n ; k_{0}}\right)$. For example, $p_{13}^{*}$ can be computed by means of $s^{3}=s^{*}\left(s^{1}, w\right)$ for $w \in B_{3 ; 3}^{*}\left(s^{1}, s^{3}\right)=\left\{(0,0,2)^{T},(1,0,2)^{T}\right.$, $(0,1,2)^{T},(1,1,2)^{T},(2,0,2)^{T},(0,2,2)^{T},(2,2,2)^{T},(1,2,2)^{T}$, $\left.(2,1,2)^{T},(2,2,3)^{T}\right\}$ and we obtain $p_{13}^{*}=\sum_{w \in B_{3 ; 3}^{*}\left(s^{1}, s^{3}\right)} q(w)=\frac{10}{64}$. Further enumerations yield $P^{*}=\frac{1}{64}\left(\begin{array}{ccc}21 & 33 & 10 \\ 3 & 49 & 12 \\ 3 & 27 & 34\end{array}\right)$. 
The computation of PRMs by means of this simple enumeration is a laborious method. No formulas are known for most of the elements in PRMs. ${ }^{8} \mathrm{We}$ will calculate and after that use limits of PRMs in the following sections.

\section{Limits of PRMs}

In this section limits of elements of PRMs are determined as $n \rightarrow \infty$ and $k_{0} \rightarrow \infty$ if the numbers of rows and columns of the corresponding PRMs are fixed. (Only limits as $s u \rightarrow \infty$ are not possible since $s u$ is limited by $n k_{0}$, see (8).)

At first, we give classes of sets of (restricted) partitions with an infinite number of sets $S_{n ; s u ; k_{0}}$ where all sets $S_{n ; s u ; k_{0}}$ have the same number of partitions so that the corresponding PRMs all have the same numbers of rows and columns.

- Sets $S_{n ; s u ; k_{0}}$ with $n>s u$ are called sets of sparse partitions.

- Sets $S_{n ; s u ; k_{0}}$ with $\overline{s u}<k_{0}$ and $\overline{s u}<n$ are called sets of non-truncated heavy partitions, where

$$
\overline{s u}:=n k_{0}-s u .\left(0<\overline{s u} \leq(n-1) k_{0}\right. \text { follows from (8).) }
$$

- Sets $S_{n ; s u ; k_{0}}$ with $\overline{s u}<k_{0}$ and $\overline{s u} \geq n$ are called sets of truncated heavy partitions.

${ }^{9}$ Obviously, sets of sparse partitions $S_{n ; s u ; k_{0}} \in\left\{S_{n ; s u ; k_{0}} \mid n>s u\right\}$ have the same number $r$ of partitions (for arbitrary but fixed $s u$ and $k_{0}$ ). This means that the corresponding PRMs are all of the same size, $r$ by $r$. Analogous statements are right for sets of non-truncated heavy partitions $S_{n ; s u ; k_{0}} \in\left\{S_{n ; s u ; k_{0}} \mid \overline{s u}<\right.$ $\left.k_{0}, \overline{s u}<n\right\}$ (for arbitrary but fixed $\overline{s u}$ ) and for sets $S_{n ; s u ; k_{0}} \in\left\{S_{n ; s u ; k_{0}} \mid \overline{s u}<\right.$ $\left.k_{0}\right\}$ (for arbitrary but fixed $\overline{s u}$ and $n(\leq \overline{s u})$ ).

\subsection{Limits of PRMs with Regard to Sets of Sparse Partitions}

Definition 6. (a) Let $s \in S_{n ; s u ; k_{0}}$ be a partition with $n_{(=)}^{>} s u$ and (w. l. o. g.) $s_{1} \geq s_{2} \geq \cdots \geq s_{n}$. Then $s_{H}=\left(s_{1}, \cdots, s_{\eta}\right)$ with $s_{i}>1$ for $i=1, \cdots, \eta$ and $s_{i} \in\{0,1\}$ for $i=\eta+1, \cdots, n$ is called the principal part of s.

(b) Let $s_{H}^{l}$ be the principal parts of the partitions $s^{l} \in S_{n_{l} ; s u_{l} ; k_{0 l}}$ (where

\footnotetext{
${ }^{8} \mathrm{~A}$ polynomial and sometimes an exponential dependence of the elements of PRMs on the variables $k_{0}$ and $n$ has been shown by means of "perturbed partitions". This will be the content of a next paper.

${ }^{9} \mathrm{~A}$ more detailed classification of sets of restricted partitions can be found in [5].
} 
$n_{l} \underset{(=)}{>} s u_{l}, \quad s_{1}^{l} \geq s_{2}^{l} \geq \cdots \geq s_{n_{l}}^{l}$ ) for $l=1,2$. Then $s^{2}$ is called a monotone successor of $s^{1}$ if $\eta_{1} \geq \eta_{2}$ and $s_{i}^{1} \geq s_{i}^{2}$ for

$i=1, \cdots, \eta_{2}\left(\eta_{1}\right)$.

Theorem 7. (Limits of PRMs with regard to sets of sparse partitions) Let $n_{(=)}^{>} s u$ and $\left\{s^{f}(n), s^{l}(n)\right\} \subseteq S_{n ; s u ; k_{0}}$ with (w. 1. o. g.) $s^{\lambda}(n)_{1} \geq s^{\lambda}(n)_{2} \geq \cdots \geq s^{\lambda}(n)_{n}$ for $\lambda=f, l$. (This means, in particular, $s^{\lambda}(n)_{s u+1}=s^{\lambda}(n)_{s u+2}=\cdots=s^{\lambda}(n)_{n}=0$ for $\lambda=f, l$.) Furthermore, let $s^{\lambda}:=\left(s^{\lambda}(n)_{1}, \cdots, s^{\lambda}(n)_{s u}\right)^{T} \in S_{s u ; s u ; k_{0}}$ for $\lambda=f, l$. Then, $\lim _{n \rightarrow \infty} p^{*}\left(s^{l}(n) \mid s^{f}(n)\right)=$

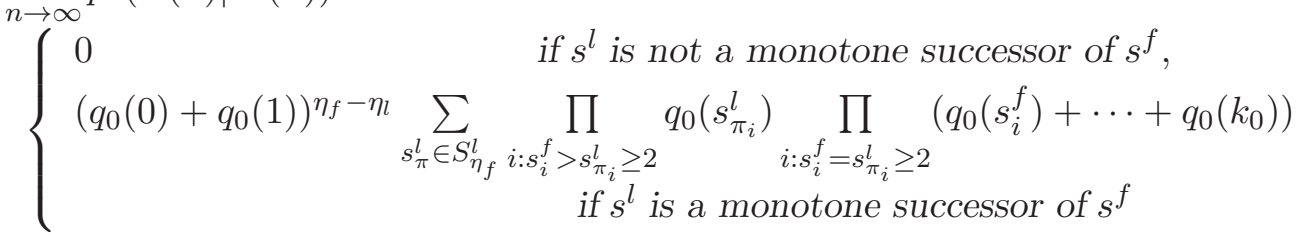

where $\eta_{f}$ and $\eta_{l}$ are defined as in Definition 6(b) and $S_{\eta_{f}}^{l}=\left\{s_{\pi} \in \mathbb{Z}_{+}^{\eta_{f}} \mid s_{\pi}\right.$ is a permutation of

$\left(s_{1}^{l}, s_{2}^{l}, \cdots, s_{\eta_{l}}^{l}, 0, \cdots, 0\right)^{T} \in \mathbb{Z}_{+}^{\eta_{f}}$ with $s_{i}^{f} \geq s_{\pi_{i}}$ for $\left.i=1, \cdots, \eta_{f}\right\}$.

Proof. Let us denote

$$
p^{*}\left(s^{l}(n) \mid s^{f}(n)\right)=\sum_{w: s^{*}\left(s^{f}(n), w\right)=s^{l}(n)} q(w)=\sum_{w \in B^{1}} q(w)+\sum_{w \in B^{2}} q(w)
$$

where $B^{1}=\left\{w \in B_{n ; k_{0}} \mid C[w ; s u] \wedge s^{*}\left(s^{f}(n), w\right)=s^{l}(n)\right\}$

and $B^{2}=\left\{w \in B_{n ; k_{0}} \mid C[s u ; w] \wedge s^{*}\left(s^{f}(n), w\right)=s^{l}(n)\right\}$.

Now, we consider $w \in B^{1}: \mathrm{C}[\mathrm{w}$; su] implies that at least $(n-s u)$ coordinates of $w$ are equal to 0 . The simple inequality relation

$$
\sum_{w \in B^{1}} q(w) \leq\left(\begin{array}{c}
n \\
s u
\end{array}\right) q_{0}(0)^{n-s u}
$$

follows and furthermore

$$
\lim _{n \rightarrow \infty} \sum_{w \in B^{1}} q(w) \leq \lim _{n \rightarrow \infty}\left(\begin{array}{c}
n \\
s u
\end{array}\right) q_{0}(0)^{n-s u}=0
$$

since $\left(\begin{array}{c}n \\ s u\end{array}\right)$ is a polynomial (of the degree $s u$ ) and $q_{0}(0)<1$ (see $(10)$ ).

Thus, $\lim _{n \rightarrow \infty} p^{*}\left(s^{l}(n) \mid s^{f}(n)\right)=\lim _{n \rightarrow \infty} \sum_{w \in B^{2}} q(w)$ remains to be considered:

Case 1: Let $s^{l}$ not be a monotone successor of $s^{f}$.

This means $\exists i: s^{l}(n)_{i}>s^{f}(n)_{i} \wedge s^{l}(n)_{i}>1$. Thus, at least $(n-s u)$ 
coordinates of $w \in B^{2}$ must be equal to 0 according to the iterative method from Section 5, case C[su, w]. Otherwise in certain iteration steps 0's would needlessly be increased to 1 and the above $s^{l}(n)_{i}$ could then not be obtained by the iterative method. Analogously to $\left({ }^{*} 1\right)$ and $\left({ }^{*} 2\right)$

$\lim _{n \rightarrow \infty} p^{*}\left(s^{l}(n) \mid s^{f}(n)\right)=\lim _{n \rightarrow \infty} \sum_{w \in B^{2}} q(w)=0$ follows in case 1 .

Case 2: Let $s^{l}$ be a monotone successor of $s^{f}$.

We partition $B^{2}=B^{2 a} \cup B^{2 b}$ where $B^{2 a}=\left\{w \in B^{2} \mid \forall\right.$ permutations $s_{\pi}^{l}(n)$ of $s^{l}(n)$ satisfying (11) $\left.\exists i: s_{\pi}^{l}(n)_{i}>s^{f}(n)_{i} \wedge s_{\pi}^{l}(n)_{i}>1\right\}$ $B^{2 b}=\left\{w \in B^{2} \mid \exists\right.$ a permutation $s_{\pi}^{l}(n)$ of $s^{l}(n)$ satisfying (11) : $s_{\pi}^{l}(n)_{i} \leq s^{f}(n)_{i}$ for $i=1, \cdots, \eta_{f}$ and
$s_{\pi}^{l}(n)_{i} \in\{0,1\}$ for $\left.i=\eta_{f}+1, \cdots, n\right\}$.

We see at first, analogous to case 1, that $\lim _{n \rightarrow \infty} \sum_{w \in B^{2 a}} q(w)=0$ follows. Finally, let $s_{\pi}^{l}(n)$ be a permutation of $s^{l}(n)$ with $s_{\pi}^{l}(n)_{i} \leq s^{f}(n)_{i}$ for $i=1, \cdots, \eta_{f}$ and $s_{\pi}^{l}(n)_{i} \in\{0,1\}$ for $i=\eta_{f}+1, \cdots, n$. Then $s^{*}\left(s^{f}(n), w\right)=s^{l}(n)$, where $s_{\pi}^{l}(n)$ together with $w$ and $s^{f}(n)$ fullfil (11), is only valid for $w \in B_{n ; k_{0}}$ with

$$
\begin{array}{ll}
s_{i}^{f} \leq w_{i} \leq k_{0} & \text { if } \quad s_{\pi}^{l}(n)_{i}=s_{i}^{f}\left(=s^{f}(n)_{i}\right) \geq 2, \\
w_{i}=s_{\pi}^{l}(n)_{i} & \text { if } \quad s_{i}^{f}>s_{\pi}^{l}(n)_{i} \geq 2 \\
w_{i} \in\{0,1\} & \text { if } \quad s_{i}^{f} \geq 2 \wedge s_{\pi}^{l}(n)_{i} \in\{0,1\} \\
0 \leq w_{i} \leq k_{0} & \text { if } \quad i>\eta_{f}
\end{array}
$$

where, additionally, $\sum_{i=1}^{n} w_{i} \geq s u$. If $w$ fullfils (19) however $\sum_{i=1}^{n} w_{i}<s u$ that implies that at least $(n-s u)$ coordinates of $w$ are equal to 0 . Analogous to $\left(*^{*}\right)$ and $\left(*^{2}\right)$ the relationship

$$
\lim _{n \rightarrow \infty} \sum_{w: \sum_{i} w_{i}<s u} q(w) \leq \lim _{n \rightarrow \infty}\left(\begin{array}{c}
n \\
s u
\end{array}\right) q_{0}(0)^{n-s u}=0
$$

follows. If $s_{\pi}^{l}(n)$ corresponds to $w$ as in $B^{2 b}$ then, obviously, $s_{\pi}^{l} \in S_{\eta_{f}}^{l}$, where $s_{\pi_{i}}^{l}=\left\{\begin{array}{ll}s_{\pi}^{l}(n)_{i} & \text { if } s_{\pi}^{l}(n)_{i} \geq 2, \\ 0 & \text { otherwise }\end{array}\right.$ for $i=1, \cdots, \eta_{f}$.

Different $s_{\pi}^{l}$ imply different $w$ which together with the corresponding $s_{\pi}^{l}(n)$ satisfy (19). This in conjunction with $(* 3)$ yields $\lim _{n \rightarrow \infty} \sum_{w \in B^{2 b}} q(w)=$ 


$$
\begin{aligned}
& \sum_{s_{\pi}^{l} \in S_{\eta_{f}}^{l}}\left(q_{0}(0)+q_{0}(1)\right)^{\eta_{f}-\eta_{l}} \prod_{i: s_{i}^{f}>s_{\pi_{i}}^{l} \geq 2} q_{0}\left(s_{\pi_{i}}^{l}\right) \prod_{i: s_{i}^{f}=s_{\pi_{i}}^{l} \geq 2}\left(q_{0}\left(s_{i}^{f}\right)+\cdots+q_{0}\left(k_{0}\right)\right)= \\
& \lim _{n \rightarrow \infty} p^{*}\left(s^{l}(n) \mid s^{f}(n)\right) .
\end{aligned}
$$

Corollary 8. Let $s u$ and $k_{0}\left(s u \geq k_{0}\right)$ be given and let the numbering of the partitions of the sets $S_{n ; s u ; k_{0}}=\left\{s^{1}(n), \cdots, s^{r}(n)\right\}$,

$n \underset{(=)}{>}$ su be so that if $s^{f}(n)$ is a (direct) predecessor of $s^{l}(n)$ then $f<l$. Then the matrix $\lim _{n \rightarrow \infty} P^{*}(n)$ is a triangular matrix.

\subsection{Limits of PRMs with Regard to Sets of Non-Truncated Heavy Partitions}

An infinite number of sets of non-truncated heavy partitions with a given fixed number of partitions is yielded by $k_{0} \rightarrow \infty$ or $n \rightarrow \infty$.

The case $n \rightarrow \infty$ can be discussed analogously to Section 6.1 (if we use $s=\left(k_{0}, \cdots, k_{0}\right)^{T}-\bar{s}$, where $s$ are non-truncated heavy partitions and $\bar{s}$ are corresponding sparse partitions.) Here we consider the case $k_{0} \rightarrow \infty$. This requires additional properties of the probability functions $q$ : Let $q^{k_{0}}$ denote probability functions corresponding to $B_{n ; k_{0}}$ (where $n$ is fixed). Then we assume

$$
\begin{gathered}
\lim _{k_{0} \rightarrow \infty} q_{0}^{k_{0}}\left(w_{i}\right)=0 \text { for } w_{i}=0,1, \ldots \text { and } \\
\exists c\left(k_{0}\right)\left(\text { with } 1>c\left(k_{0}\right)>0\right): \exists \lim _{k_{0} \rightarrow \infty} \frac{q_{0}^{k_{0}}\left(w_{i}\right)}{c\left(k_{0}\right)} \neq 0 \text { for } w_{i}=0,1, \ldots \\
\lim _{k_{0} \rightarrow \infty} \frac{\left(q_{0}^{k_{0}}\left(w_{i}\right)\right)^{l}}{c\left(k_{0}\right)}=0 \text { for } l \geq 2 \text { and } w_{i}=0,1, \ldots
\end{gathered}
$$

follows from (20) and (21). (The limits $\lim _{k_{0} \rightarrow \infty} \frac{q_{0}^{k_{0}}(\cdot)}{c\left(k_{0}\right)}$ are unique, however can differ by a constant multiple (in relation to $\left.c\left(k_{0}\right)\right)$.)

Definition 9. (a) Let $s \in S_{n ; s u ; k_{0}}$ be a non-truncated heavy partition with (w. l. o. g.) $s_{1} \geq s_{2} \geq \cdots \geq s_{n}$. Then $s_{H}=\left(s_{\eta}, \cdots, s_{n}\right)$ with $s_{i}<k_{0}-1$ for $i=\eta, \cdots, n$ and $s_{i} \in\left\{k_{0}, k_{0}-1\right\}$ for $i=1, \cdots, \eta-1$ is called the principal part of $s$.

(b) Let $s_{H}^{l}$ be the principal parts of non-truncated heavy partitions $s^{l} \in$ $S_{n_{l} ; s u_{l} ; k_{0_{l}}}$ (where $s_{1}^{l} \geq s_{2}^{l} \geq \cdots \geq s_{n_{l}}^{l}$ ) for $l=1,2$. Then $s^{2}$ is called a restricted monotone successor of $s^{1}$ if $n_{1}-\eta_{1} \geq n_{2}-\eta_{2}$, and $s_{n_{1}-i}^{1} \leq$ 
$s_{n_{2}-i}^{2}$ for $i=0, \cdots,\left(n_{1}-\eta_{1}\right) n_{2}-\eta_{2}$ and

a permutation $s_{\pi}^{2}$ of $s^{2}$ exists such that $s_{n_{1}-i}^{1} \neq s_{\pi\left(n_{2}-i\right)}^{2}$

for at most one $i \in\left\{0, \cdots, n_{1}-\eta_{1}\right\}$.

(This also implies $n_{1}-\eta_{1}=n_{2}-\eta_{2}$ or $n_{1}-\eta_{1}=n_{2}-\eta_{2}+1$.)

Theorem 10. (Limits of PRMs with regard to sets of non-truncated heavy partitions) Let $S_{n ; s u ; k_{0}}$ be sets of non-truncated heavy partition where su is represented by $s u=n k_{0}-\overline{s u}$ with fixed $n$ and $\overline{s u}$ (and $n \geq 2, k_{0} \geq 2$ ). Furthermore, let $\left\{s^{f}\left(k_{0}\right), s^{l}\left(k_{0}\right)\right\} \subseteq S_{n ; s u ; k_{0}}$ with (w. l. o. g.) $s^{\lambda}\left(k_{0}\right)_{1} \geq$ $s^{\lambda}\left(k_{0}\right)_{2} \geq \cdots \geq s^{\lambda}\left(k_{0}\right)_{n}$ for $\lambda=f ; l$. (In particular, that means $s^{\lambda}\left(k_{0}\right)_{1}=$ $\cdots=s^{\lambda}\left(k_{0}\right)_{n-s \bar{u}}=k_{0}$ for $\lambda=f ; l$. $)$ In addition let $s^{\lambda}=\left(s_{1}^{\lambda}, \cdots, s_{s \bar{u}}^{\lambda}\right)^{T}:=$ $\left(s^{\lambda}\left(k_{0}\right)_{n-s \bar{u}+1}, \cdots, s^{\lambda}\left(k_{0}\right)_{n}\right)^{T} \in S_{\overline{s u}, s u, k_{0}}$ (where $\left.\sum_{i} s_{i}^{\lambda}=\overline{s u} k_{0}-\overline{s u}\right)$. Finally, let given probability functions $q^{k_{0}}$ fulfill (20) and (21) for certain $c\left(k_{0}\right)$ and $q_{0}^{0}\left(w_{i}\right):=\lim _{k_{0} \rightarrow \infty} \frac{q_{0}^{k_{0}}\left(w_{i}\right)}{c\left(k_{0}\right)}$. Then,

$$
\begin{aligned}
& \lim _{k_{0} \rightarrow \infty} \frac{1}{c\left(k_{0}\right)}\left(p^{*}\left(s^{l}\left(k_{0}\right) \mid s^{f}\left(k_{0}\right)\right)-\delta\left(s^{l}\left(k_{0}\right), s^{f}\left(k_{0}\right)\right)\right) \\
& =\left\{\begin{array}{c}
\begin{array}{c}
\text { if } s^{l} \text { is not a restricted monotone successor of } s^{f}, \\
-\sum_{i: s_{i}^{f} \leq k_{0}-2}\left(q_{0}^{0}\left(s_{i}^{f}+1\right)+\cdots+q_{0}^{0}\left(k_{0}\right)\right)
\end{array} \\
\sum_{s_{\pi}^{l} \in S_{\eta_{f}}^{l}}\left\{\begin{array}{l}
q_{0}^{0}\left(s_{\pi_{i_{0}}}^{l}\right) \\
\left(q_{0}^{0}\left(k_{0}-1\right)+q_{0}^{0}\left(k_{0}\right)\right) \text { for } s_{\pi_{i_{0}}}^{l}=s_{i_{0}}=k_{0}-1, \\
\text { if } s^{l} \text { is a restricted monotone successor of } s^{f} \text { and } s^{l} \neq s^{f}
\end{array}\right.
\end{array}\right.
\end{aligned}
$$

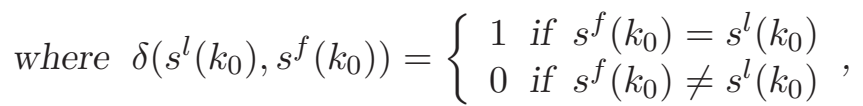

$$
\begin{aligned}
& S_{\eta_{f}}^{l}=\left\{s_{\pi} \in \mathbb{Z}_{+}^{n-\eta_{f}+1} \mid s_{\pi}\right. \text { is a permutation } \\
& \text { of }\left\{\begin{array}{ll}
\left(s_{\eta_{l}}^{l}, \cdots, s_{n}^{l}\right)^{T} & \text { if } \eta_{f}=\eta_{l}, \\
\left(k_{0}-1, s_{\eta_{l}}^{l}, \cdots, s_{n}^{l}\right)^{T} & \text { if } \eta_{f}=\eta_{l}-1
\end{array} \text { with } s_{i}^{f} \leq s_{\pi_{i}}\right. \\
& \text { for } \left.i=\eta_{f}, \cdots, n \text { and } s_{i_{0}}^{f} \neq s_{\pi_{i_{0}}} \text { for one } i_{0}\right\}
\end{aligned}
$$

and $\eta_{f}, \eta_{l}$ are as in Definition $9(b)$.

Formally, the proof is analogous to the proof of Theorem 7, however the roles of the cases $C[w, s u]$ and $C[s u, w]$ are reversed. In detail the considerations are

\footnotetext{
${ }^{10}$ This is a finite number of i since $s \bar{u}$ is fixed.
} 
somewhat different. We demonstrate this for $\sum_{w \in B^{2}} q^{k_{0}}(w)$ (where $B^{2}=\{w \in$ $\left.\left.B_{n ; k_{0}} \mid C[s u ; w] \wedge s^{*}\left(s^{f}\left(k_{0}\right), w\right)=s^{l}\left(k_{0}\right)\right\}\right)$. (11), case C $[\mathrm{su} ; \mathrm{w}]$ implies that an $s_{\pi}^{l}\left(k_{0}\right)$, a permutation of $s^{l}\left(k_{0}\right)$, exists with $w \geq s_{\pi}^{l}\left(k_{0}\right)$. Hence, at least one coordinate of $w$ is equal to $k_{0}$ and at least one is either also equal to $k_{0}$ or is equivalent to $k_{0}-1$ ( since $s_{\pi}^{l}\left(k_{0}\right)$ is a non-truncated heavy partition). The simple inequality relation

$\frac{1}{c\left(k_{0}\right)} \sum_{w \in B^{2}} q^{k_{0}}(w) \leq \frac{1}{c\left(k_{0}\right)}\left(\begin{array}{c}n \\ 2\end{array}\right)\left(q_{0}^{k_{0}}\left(k_{0}\right)+q_{0}^{k_{0}}\left(k_{0}-1\right)\right)^{2}$

follows and furthermore (22) yields

$$
\sum_{w \in B^{2}} q_{0}^{0}(w) \leq \lim _{k_{0} \rightarrow \infty} \frac{1}{c\left(k_{0}\right)}\left(\begin{array}{c}
n \\
2
\end{array}\right)\left(q_{0}^{k_{0}}\left(k_{0}\right)+q_{0}^{k_{0}}\left(k_{0}-1\right)\right)^{2}=0 .
$$

Corollary 11. Let sets of non-truncated heavy partition $S_{n ; s u ; k_{0}}$ as in Theorem 10 be given and let the numbering of the partitions of the sets $S_{n ; s u ; k_{0}}=\left\{s^{1}\left(k_{0}\right), \cdots, s^{r}\left(k_{0}\right)\right\}$ be such that if $s^{f}\left(k_{0}\right)$ is a (direct) predecessor of $s^{l}\left(k_{0}\right)$ then $f<l$. Then, the matrix $\lim _{k_{0} \rightarrow \infty} \frac{1}{c\left(k_{0}\right)}\left(P^{*}\left(k_{0}\right)-I\right)$ is a triangular matrix.

These limits as well as the formulas for limits of PRMs with regard to sets of sparse partitions (Theorems 7) and of PRMs with regard to sets of non-truncated heavy partitions (Theorems 10) are very different. In contrast to that, solutions of Poisson equations with regard to limits of corresponding PRMs will have similar structures, see the Theorems 15 and 19.

\section{The Monotonicity of Solutions of the Poisson Equations}

It is simple to prove the conjecture (Con") for a "small" number of certain Poisson equations where the corresponding PRMs and the right sides of the equations satisfy the "conditions of dominance". The main results of this section are the proofs of the conjecture in relation to PRMs, which are based on sets of sparse partitions with sufficiently great $n$ or on sets of non-truncated heavy partitions with sufficiently great $k_{0}$. Limits of PRMs with regard to sets of sparse partitions and sets of non-truncated heavy partitions are used for these proofs. The solutions of the Poisson equations with regard to limits of PRMs have an elegant structure, in contrast to the formulas of the limits of PRMs themselves. 


\subsection{Partial Results}

Definition 12. Let a set of restricted partitions $S_{n ; s u ; k_{0}}=\left\{s^{1}, s^{2}, \cdots, s^{r}\right\}$ be given. If $s^{f}$ is a direct predecessor of $s^{l}$ if and only if $f=l-1$ for $f=1,2, \cdots, r-1$ then this set of restricted partitions is called m-totally ordered.

Theorem 13. Let a m-totally ordered set of restricted partitions $S_{n ; s u ; k_{0}}=$ $\left(\left\{s^{1}, s^{2}, \cdots, s^{r}\right\}\right.$ and the corresponding PRM $P^{*}$ be given. Then the dominance condition

$$
\sum_{l=1}^{\bar{l}} p_{1 l}^{*} \geq \sum_{l=1}^{\bar{l}} p_{2 l}^{*} \geq \cdots \geq \sum_{l=1}^{\bar{l}} p_{r l}^{*} \text { for } \bar{l}=1,2, \cdots, r
$$

is fulfilled for $P^{*}$ and the solutions of the corresponding Poisson equations are monotone (in $\nu$ ).

The proof of Theorem 13 can be found in [4], Lemma 3.26 and Corollary 3.27. (In general, PRMs do not satisfy the dominance property (24)). The following Theorem 14 results from detailed computations.

Theorem 14. Solutions of Poisson equations are in general monotone for PRMs with regard to sets of restricted partitions with at most 3 partitions and with regard to sets with 4 partitions in the case of discrete uniformly distributed requirements.

\subsection{The Poisson Equations with Regard to Sets of Sparse Partitions with Sufficiently Large $n$}

In this section we consider equation systems similar to the Poisson equations where PRMs with regard to sets of sparse partitions are initially replaced by the limits of such matrices, as $n$ approaches infinity. The solutions of such equation systems will be vectors which include, in relation to the distribution of requirements, generalized harmonic numbers. The solutions are also monotone, from which the monotonicity of the solutions of the Poisson equations themselves, with regard to sets of sparse partitions for sufficiently great $n$, follows. In the considerations in this subsection, we also use the definition of monotone successors and principle parts of partitions (Definition 6) as we have used these for the computation of the limits of PRMs with regard to sets of sparse partitions in Section 6.1. Since monotone successors and principle parts do not directly depend on $s u$ and $k_{0}$ we can include all equation systems (in relation to different $s u$ and $k_{0}$ ) in one proof. Now, we give a affine transformation of 
$\gamma$, which directly depends only on the principal parts of the partitions and the distribution of the requirements:

$$
\begin{aligned}
\gamma(s) & =\sum_{i=1}^{n} \sum_{w_{i}=0}^{s_{i}}\left(s_{i}-w_{i}\right) q_{0}\left(w_{i}\right) \quad(\text { see }(16)) \\
& =\sum_{i=1}^{\eta} \sum_{w_{i}=0}^{s_{i}}\left(s_{i}-w_{i}\right) q_{0}\left(w_{i}\right)+\left(s u-\sum_{i=1}^{\eta} s_{i}\right) q_{0}(0) \\
& =\sum_{i: s_{i} \geq 2} \sum_{w_{i}=\mathbf{1}}^{s_{i}}\left(s_{i}-w_{i}\right) q_{0}\left(w_{i}\right)+s u q_{0}(0)
\end{aligned}
$$

with $\eta$ as in Definition 6(a) and $s u q_{0}(0)$ is independent of s. We define

$$
\begin{aligned}
& \gamma^{\prime}(s):=\sum_{i: s_{i} \geq 2} \sum_{w_{i}=1}^{s_{i}}\left(s_{i}-w_{i}\right) q_{0}\left(w_{i}\right)=\sum_{i: s_{i} \geq 2} \sum_{w_{i}=1}^{s_{i}-1}\left(s_{i}-w_{i}\right) q_{0}\left(w_{i}\right), \\
& \quad \text { in particular, } \gamma^{\prime}\left((1, \cdots, 1,0, \cdots, 0)^{T}\right):=0 \text { and }
\end{aligned}
$$

$$
\gamma^{\prime}\left(s_{i}\right):=\sum_{w_{i}=1}^{s_{i}}\left(s_{i}-w_{i}\right) q_{0}\left(w_{i}\right) \quad \text { for } s_{i} \geq 2 .
$$

If we now consider the equation systems

$$
g(-1, \cdots,-1)^{T}+\left(\lim _{n \rightarrow \infty} P^{*}(n)-I\right) \nu=-\gamma^{\prime} \text { with } \gamma^{\prime} \text { from }(26)
$$

then $g=0$ initially follows from the last equation (see also Corollary 8), and we see in the following theorem that the solutions $\nu\left(=\nu(s), s \in S_{n ; s u ; k_{0}}\right)$ only depend on the principal parts of the partitions and the distributions of the requirements. The sets $S_{n ; s u ; k_{0}}$ themselves are therefore insignificant for the calculations.

Theorem 15. Let $S_{n ; s u ; k_{0}}, n=(s u) s u+1,, s u+2, \cdots$ be sets of sparse partitions, each of these sets with $r$ partitions and with the partial dominance ordering. Let $B_{n ; k_{0}}$ be corresponding sets of requirements with the same marginal probability functions $q_{0}$ for all $n$ and where the requirements $w_{i},(i=1, \cdots, n)$ are independent and identically distributed and where (10) is additionally assumed. Furthermore, let $P^{*}(n)$ be the the corresponding PRMs. Then, $\nu\left((1,1, \cdots, 1,0,0, \cdots, 0)^{T}\right):=0, \nu(s)=$

$$
\begin{array}{r}
\sum_{i: s_{i} \geq 2}\left(\frac{q_{0}(1)}{q_{0}(0)+q_{0}(1)}+\frac{q_{0}(1)+q_{0}(2)}{q_{0}(0)+q_{0}(1)+q_{0}(2)}+\cdots+\frac{q_{0}(1)+q_{0}(2)+\cdots+q_{0}\left(s_{i}-1\right)}{q_{0}(0)+q_{0}(1)+\cdots+q_{0}\left(s_{i}-1\right)}\right), \\
s \in S_{n ; s u ; k_{0}}, s \neq(1,1, \cdots, 1,0,0, \cdots, 0)^{T}
\end{array}
$$




$$
\begin{gathered}
=\sum_{i: s_{i} \geq 2}\left(s_{i}-q_{0}(0)\left(\frac{1}{Q_{0}(0)}+\frac{1}{Q_{0}(1)}+\cdots+\frac{1}{Q_{0}\left(s_{i}-1\right)}\right)\right) \text { where } \\
Q_{0}(\omega)=q_{0}(0)+q_{0}(1)+\cdots+q_{0}(\omega) \text { together with a }
\end{gathered}
$$

corresponding value of $g$ are solutions of the equation system (27).

Briefly, we note that $\frac{1}{Q_{0}(0)}+\frac{1}{Q_{0}(1)}+\cdots+\frac{1}{Q_{0}\left(S_{i}-1\right)}$ are, in relation to the distribution of requirements, generalized harmonic numbers.

Proof. 1. We use the following notations and definitions

$$
\begin{aligned}
& Q_{0}(\omega):=q_{0}(0)+q_{0}(1)+\cdots+q_{0}(\omega), \\
& Q_{1}(\omega):=\quad q_{0}(1)+\cdots+q_{0}(\omega)\left(=Q_{0}(\omega)-q_{0}(0)\right), \\
& \bar{Q}_{0}(\omega):=q_{0}(\omega)+q_{0}(\omega+1)+\cdots+q_{0}\left(k_{0}\right)\left(=1-Q_{0}(\omega-1)\right), \\
& \nu\left(s_{i}\right):=\sum_{\omega=1}^{s_{i-1}} \frac{Q_{1}(\omega)}{Q_{0}(\omega)} \text { for } s_{i} \geq 2, \nu(0):=0, \nu(1):=0
\end{aligned}
$$

(hence $\left.\nu(s)=\sum_{i: s_{i} \geq 2} \nu\left(s_{i}\right)=\sum_{i=1}^{n} \nu\left(s_{i}\right)\right)$,

$\wp(M)$ - the power set of a set $M, \wp:=\wp(\{1,2, \cdots, \eta\})$,

$\wp^{-}:=\wp \backslash\{1,2, \cdots \eta\}, \wp_{j}:=\wp(\{1,2, \cdots, \eta\} \backslash\{j\})$,

$M S(s):=\left\{s^{2} \in S_{n ; s u ; k_{0}} \mid s^{2}\right.$ is a monotone successor of $\left.s\right\}$.

Furthermore, we note that if $\bar{I} \in \wp$ then $\{1,2, \cdots, \eta\} \backslash \bar{I}:=\left\{j_{1}, j_{2}, \cdots, j_{h}\right\}$ with $h=\eta-|\bar{I}|$.

2. Now, we give 4 relationships which are employed in the following proof:

Without loss of generality, let be $s_{i} \geq 2$ for $i=1,2, \cdots, \eta$ and $s_{j} \in\{0,1\}$ for $j=\eta+1, \eta+2, \cdots, n$.

$$
\begin{aligned}
1 & =\sum_{l=1}^{r} \lim _{n \rightarrow \infty} p^{*}\left(s^{l} \mid s\right) \\
= & \sum_{s^{2} \in M S(s)}\left(q_{0}(0)+q_{0}(1)\right)^{\eta-\eta_{2}} \sum_{s_{\pi}^{2} \in S_{\pi}^{2}}\left(\prod_{i: s_{i}>s_{\pi_{i}}^{2} \geq 2} q_{0}\left(s_{\pi_{i}}^{2}\right)\right)\left(\prod_{i: s_{i}=s_{\pi_{i}}^{2} \geq 2} \bar{Q}_{0}\left(s_{i}\right)\right)
\end{aligned}
$$

(see Theorem 7) These summations over all monotone successors of $s$ can however be realized in the following way:

$$
\begin{aligned}
& =\sum_{\bar{I} \in \wp}\left(\prod_{i \in \bar{I}} \bar{Q}_{0}\left(s_{i}\right)\right) \sum_{s_{\pi_{j_{1}}}^{2}=0}^{s_{j_{1}}-1} \ldots \sum_{s_{\pi_{j_{h}}}^{2}=0}^{s_{j_{h}}-1} \prod_{h^{\prime}=1}^{h} q_{0}\left(s_{\pi_{j_{h^{\prime}}}}^{2}\right) \text { (which means } \\
& \left.s_{i}=s_{\pi_{i}}^{2} \geq 2 \text { for } i \in \bar{I} \text { and } s_{j}>s_{\pi_{j}}^{2} \wedge s_{j} \geq 2 \text { for } j \in\{1,2, \cdots, \eta\} \backslash \bar{I}\right) \\
& =\sum_{\bar{I} \in \wp}\left(\prod_{i \in \bar{I}} \bar{Q}_{0}\left(s_{i}\right)\right)\left(\prod_{j \notin \bar{I}} Q_{0}\left(s_{j}-1\right) \text { where } \prod_{i \in \bar{I}} \bar{Q}_{0}\left(s_{i}\right):=1\right. \\
& \quad \text { for } \bar{I}=\varnothing, \prod_{j \notin \bar{I}} Q_{0}\left(s_{j}-1\right):=1 \text { for } \bar{I}=\{1,2, \cdots, \eta\} . \\
& \sum_{\bar{I} \in \wp^{-}}\left(\prod_{i \in \bar{I}} \bar{Q}_{0}\left(s_{i}\right)\right)\left[\sum_{j \notin \bar{I}}\left(\prod_{j^{\prime} \notin \bar{I} \cup\{j\}} Q_{0}\left(s_{j^{\prime}}-1\right)\right) \gamma^{\prime}\left(s_{j}\right)\right]
\end{aligned}
$$




$$
\begin{gathered}
=\sum_{j=1}^{\eta}\left[\gamma^{\prime}\left(s_{j}\right) \sum_{\bar{I} \in \wp_{j}}\left(\prod_{i \in \bar{I}} \bar{Q}_{0}\left(s_{i}\right)\right)\left(\prod_{j^{\prime} \notin \bar{I} \cup\{j\}} Q_{0}\left(s_{j^{\prime}}-1\right)\right)\right] \\
=\sum_{j=1}^{\eta} \gamma^{\prime}\left(s_{j}\right) \cdot 1 \quad \text { (see }(* 1), \text { applied to } s \text { with the principal } \\
=\gamma^{\prime}(s) . \\
\left.\operatorname{parts}\left(s_{1}, \cdots, s_{j-1}, s_{j+1}, \cdots, s_{\eta}\right)\right) \\
\lim _{n \rightarrow \infty} p^{*}(s \mid s)=\prod_{i: s_{i} \geq 2}\left(q_{0}\left(s_{i}\right)+\cdots+q_{0}\left(k_{0}\right)\right)=\prod_{i: s_{i} \geq 2} \bar{Q}_{0}\left(s_{i}\right)
\end{gathered}
$$

(see Theorem 7).

$$
\begin{aligned}
\gamma^{\prime}\left(s_{i}\right) & =\sum_{\omega=1}^{s_{i}}\left(s_{i}-\omega\right) q_{0}(\omega)=\sum_{\beta=1}^{s_{i}-1} \sum_{\omega=1}^{\beta} q_{0}(\omega) \quad \text { the sum) } \\
& =\sum_{\beta=1}^{s_{i}-1}\left(\sum_{\omega=0}^{\beta} q_{0}(\omega)\right) \frac{Q_{1}(\beta)}{Q_{0}(\beta)} \\
& =\left(\sum_{\omega=0}^{s_{i}-1} q_{0}(\omega)\right) \sum_{\beta=1}^{s_{i}-1} \frac{Q_{1}(\beta)}{Q_{0}(\beta)}-\sum_{\beta=1}^{s_{i}-2}\left(\sum_{\omega=\beta+1}^{s_{i}-1} q_{0}(\omega)\right) \frac{Q_{1}(\beta)}{Q_{0}(\beta)} \\
& =Q_{0}\left(s_{i}-1\right) \nu\left(s_{i}\right)-\sum_{\omega=2}^{s_{i}-1} q_{0}(\omega) \nu(\omega)
\end{aligned}
$$

(reorganization of the last sums; $\nu(\cdot)$ as in this theorem).

3. The proof of the Theorem: (27) together with Corollary 8 yields

$$
\nu(s)=\frac{1}{1-\lim _{n \rightarrow \infty} p^{*}(s \mid s)}\left(\gamma^{\prime}(s)+\sum_{s^{2} \in M S(s) \backslash\{s\}} \lim _{n \rightarrow \infty} p^{*}\left(s^{2} \mid s\right) \nu\left(s^{2}\right)\right) .
$$

An equivalent equation is

$$
0=\gamma^{\prime}(s)-\nu(s)+\sum_{s^{2} \in M S(s)} \lim _{n \rightarrow \infty} p^{*}\left(s^{2} \mid s\right) \nu\left(s^{2}\right)
$$

Further equivalent transformations, using the summation over all monotone successors as in the derivation of $(* 1)$, yield

$$
\begin{aligned}
0=\gamma^{\prime}(s)-\nu(s) & +\sum_{\bar{I} \in \wp}\left(\prod_{i \in \bar{I}} \bar{Q}_{0}\left(s_{i}\right)\right)\left[\sum_{s_{j_{1}}^{2}=0}^{s_{j_{1}}-1} \cdots \sum_{s_{j_{h-1}}^{2}=0}^{s_{j_{h-1}-1}-1}\right. \\
& \left.\sum_{s_{j_{h}}^{2}=0}^{s_{j_{h}}-1}\left(\prod_{h^{\prime}=1}^{h} q_{0}\left(s_{j_{h^{\prime}}}^{2}\right)\right)\left(\sum_{i \in \bar{I}} \nu\left(s_{i}\right)+\sum_{h^{\prime}=1}^{h} \nu\left(s_{j_{h^{\prime}}}^{2}\right)\right)\right]
\end{aligned}
$$

$\left(\left(q_{0}(0)+q_{0}(1)\right)^{\eta-\eta_{2}}\right.$ from Theorem 7 is realized by $s_{j_{h^{\prime}}}^{2}=0 ; 1$ for $h^{\prime}=$ $1, \cdots, h)$ where $\prod_{i \in \bar{I}} \bar{Q}_{0}\left(s_{i}\right):=1$ for $I=\varnothing$. We now apply $(* 4)$ in the form 
of $\sum_{s_{j_{h^{\prime}}}^{2}=0}^{s_{j_{h^{\prime}}}-1} q_{0}\left(s_{j_{h^{\prime}}}^{2}\right) \nu\left(s_{j_{h^{\prime}}}^{2}\right)=-\gamma^{\prime}\left(s_{j_{h^{\prime}}}\right)+Q_{0}\left(s_{j_{h^{\prime}}}-1\right) \nu\left(s_{j_{h^{\prime}}}\right)($ with $\nu(\cdot)$ as in this theorem) to the above equation (at first for $h^{\prime}=h$ ):

$$
\begin{aligned}
& 0=\gamma^{\prime}(s)-\nu(s)+\sum_{\bar{I} \in \wp}\left(\prod_{i \in \bar{I}} \bar{Q}_{0}\left(s_{i}\right)\right)\left[\sum_{s_{j_{1}}^{2}=0}^{s_{j_{1}}-1} \cdots \sum_{s_{j_{h-1}}^{2}}^{s_{j_{h-1}}-1}=0\right. \\
& {\left[\left(\prod_{h^{\prime}=1}^{h-1} q_{0}\left(s_{j_{h^{\prime}}}^{2}\right)\right) Q_{0}\left(s_{j_{h}}-1\right)\left(\sum_{i \in \bar{I}} \nu\left(s_{i}\right)+\sum_{h^{\prime}=1}^{h-1} \nu\left(s_{j_{h^{\prime}}}^{2}\right)\right)+\right.} \\
& \left.\left.\prod_{h^{\prime}=1}^{h-1} q_{0}\left(s_{j_{h^{\prime}}}^{2}\right)\left(-\gamma^{\prime}\left(s_{j_{h}}\right)+Q_{0}\left(s_{j_{h}}-1\right) \nu\left(s_{j_{h}}\right)\right)\right]\right] \\
& 0=\gamma^{\prime}(s)-\nu(s)+\sum_{\bar{I} \in \wp}\left(\prod_{i \in \bar{I}} \bar{Q}_{0}\left(s_{i}\right)\right)\left[\sum_{s_{j_{1}}^{2}=0}^{s_{j_{1}}-1} \cdots \sum_{s_{j_{h-1}}^{2}}^{s_{j_{h-1}-1}}=0\right. \\
& {\left[\left(\prod_{h^{\prime}=1}^{h-1} q_{0}\left(s_{j_{h^{\prime}}}^{2}\right)\right) Q_{0}\left(s_{j_{h}}-1\right)\left(\sum_{i \in \bar{I} U\left\{j_{h}\right\}} \nu\left(s_{i}\right)+\sum_{h^{\prime}=1}^{h-1} \nu\left(s_{j_{h^{\prime}}}^{2}\right)\right)\right.} \\
& \left.\left.-\prod_{h^{\prime}=1}^{h-1} q_{0}\left(s_{j_{h^{\prime}}}^{2}\right) \gamma^{\prime}\left(s_{j_{h}}\right)\right]\right] \text {. }
\end{aligned}
$$

The repeated application of $\left({ }^{*} 4\right)\left(\right.$ for $\left.h^{\prime}=h-1\right)$ yields

$$
\begin{aligned}
& 0=\gamma^{\prime}(s)-\nu(s)+\sum_{\bar{I} \in \wp}\left(\prod_{i \in \bar{I}} \bar{Q}_{0}\left(s_{i}\right)\right)\left[\sum _ { s _ { j _ { 1 } } ^ { 2 } = 0 } ^ { s _ { j _ { 1 } } - 1 } \cdots \sum _ { s _ { j _ { h - 2 } } ^ { 2 } = 0 } ^ { s _ { j _ { h - 2 } - 1 } } \left[\left(\prod_{h^{\prime}=1}^{h-2} q_{0}\left(s_{j_{h^{\prime}}}^{2}\right)\right)\right.\right. \\
& \prod_{h^{\prime}=h-1}^{h} Q_{0}\left(s_{j_{h^{\prime}}}-1\right)\left(\sum_{i \in \bar{I} U\left\{j_{h}\right\}} \nu\left(s_{i}\right)+\sum_{h^{\prime}=1}^{h-2} \nu\left(s_{j_{h^{\prime}}}^{2}\right)\right) \\
& -\prod_{h^{\prime}=1}^{h-2} q_{0}\left(s_{j_{h^{\prime}}}^{2}\right) Q_{0}\left(s_{j_{h-1}}-1\right) \gamma^{\prime}\left(s_{j h}\right)+\prod_{h^{\prime}=1}^{h-2} q_{0}\left(s_{j_{h^{\prime}}}^{2}\right) Q_{0}\left(s_{j_{h}}-1\right)\left(-\gamma^{\prime}\left(s_{j_{h-1}}\right)\right. \\
& \left.\left.\left.+Q_{0}\left(s_{j_{h-1}}-1\right) \nu\left(s_{j_{h-1}}\right)\right)\right]\right] \\
& 0=\gamma^{\prime}(s)-\nu(s)+\sum_{\bar{I} \in \wp}\left(\prod_{i \in \bar{I}} \bar{Q}_{0}\left(s_{i}\right)\right)\left[\sum _ { s _ { j _ { 1 } } ^ { 2 } = 0 } ^ { s _ { j _ { 1 } } - 1 } \cdots \sum _ { s _ { j _ { h - 2 } } ^ { 2 } } ^ { s _ { j _ { h - 2 } - 2 } - 1 } \left[\left(\prod_{h^{\prime}=1}^{h-2} q_{0}\left(s_{j_{h^{\prime}}}^{2}\right)\right)\right.\right. \\
& \prod_{h^{\prime}=h-1}^{h} Q_{0}\left(s_{j_{h^{\prime}}}-1\right)\left(\sum_{i \in \bar{I} U\left\{j_{h}, j_{h-1}\right\}} \nu\left(s_{i}\right)+\sum_{h^{\prime}=1}^{h-2} \nu\left(s_{j_{h^{\prime}}}^{2}\right)\right) \\
& \left.\left.-\prod_{h^{\prime}=1}^{h-2} q_{0}\left(s_{j_{h^{\prime}}}^{2}\right) Q_{0}\left(s_{j_{h-1}}-1\right) \gamma^{\prime}\left(s_{j_{h}}\right)-\prod_{h^{\prime}=1}^{h-2} q_{0}\left(s_{j_{h^{\prime}}}^{2}\right) Q_{0}\left(s_{j_{h}}-1\right) \gamma^{\prime}\left(s_{j_{h-1}}\right)\right]\right]
\end{aligned}
$$




$$
\begin{aligned}
0=\gamma^{\prime}(s)-\nu(s)+ & \sum_{\bar{I} \in \wp}\left(\prod_{i \in \bar{I}} \bar{Q}_{0}\left(s_{i}\right)\right) \prod_{j \notin \bar{I}} Q_{0}\left(s_{j}-1\right) \nu(s)- \\
& \sum_{\bar{I} \in \wp^{-}}\left(\prod_{i \in \bar{I}} \bar{Q}_{0}\left(s_{i}\right)\right)\left[\sum_{j \notin \bar{I}}\left(\prod_{j^{\prime} \notin \bar{I} U\{j\}} Q_{0}\left(s_{j^{\prime}}-1\right)\right) \gamma^{\prime}\left(s_{j}\right)\right] .
\end{aligned}
$$

Finally, (*1) implies

$$
0=\gamma^{\prime}(s)-\sum_{\bar{I} \in \wp^{-}}\left(\prod_{i \in \bar{I}} \bar{Q}_{0}\left(s_{i}\right)\right)\left[\sum_{j \notin \bar{I}}\left(\prod_{j^{\prime} \notin \bar{I} U\{j\}} Q_{0}\left(s_{j^{\prime}}-1\right)\right) \gamma^{\prime}\left(s_{j}\right)\right] \text {. }
$$

This is a valid equation, as seen by $\left(*^{*}\right)$. Thus, the assertion of the Theorem is confirmed since we have only used equivalent transformations of the equations.

Corollary 16. Let the same assumptions as in Theorem 15 be valid. Furthermore, let $s^{f}$ be a (direct) predecessor of $s^{l}$, where $\left\{s^{f}, s^{l}\right\} \subseteq S_{n ; s u ; k_{0}}$ for any $n_{(=)}^{>}$su and $s^{f} \neq s^{l}$. Then, $\nu\left(s^{l}\right)<\nu\left(s^{f}\right)$ is valid for solutions of the equation system (27). This means that the solutions of the equation systems (27) are monotone (with respect to the partial order).

This statement results from simple computations using the formulas from Theorem 15 for $\nu\left(s^{l}\right)$ and $\nu\left(s^{f}\right)$.

Corollary 17. Let the same assumptions as in Theorem 15 be valid. Then, the solutions of the Poisson equations (see (15)) with regards to sets $S_{n ; s u ; k_{0}}$ of sparse partitions are monotone for sufficiently large $n$.

This result follows from the solution behavior of linear equation systems for cases of passing to the limits in the coefficient matrices and Corollary 16 (where it is used in particular that the inequalities $\nu\left(s^{l}\right)<\nu\left(s^{f}\right)$ are strict inequalities).

Corollary 18. Let the same assumptions as in Theorem 15 be valid. This means, in particular, that sets $S_{n ; s u ; k_{0}}, n_{(=)}^{>} s u$ of sparse partitions, each of these sets with $r$ partitions, are considered. The corresponding Poisson equations, with exception of perhaps a finite number of them, then have monotone solutions.

\subsection{The Poisson Equations with Regard to Sets of Non-Truncated Heavy Partitions with Sufficiently Large $n$ or $k_{0}$}

Formally, we proceed as in Section 7.2. Differences are found in the following: In Section 7.2 we used $\lim _{n \rightarrow \infty} P^{*}(n)$ in order to show the monotonicity of 
$\nu_{f}$ (for large n), which are solutions of the equation systems (15) (see Theorem 15 and Corollary 17). We now begin with

$$
g^{\prime}(-1, \cdots,-1)^{T}+\lim _{k_{0} \rightarrow \infty} \frac{1}{c\left(k_{0}\right)}\left(P^{*}\left(k_{0}\right)-I\right) \nu=-\lim _{k_{0} \rightarrow \infty} \frac{1}{c\left(k_{0}\right)} \gamma^{\prime}
$$

(with $g^{\prime}=\lim _{k_{0} \rightarrow \infty} \frac{1}{c\left(k_{0}\right)} g, g=g\left(k_{0}\right)$ ) by reason of $(20)$.

The definition of the restricted monotone successor (Definition 9(b)) includes an additional property in comparison with Definition 6(b). Although $\lim _{n \rightarrow \infty} P^{*}(n)-I \neq \lim _{k_{0} \rightarrow \infty} \frac{1}{c\left(k_{0}\right)}\left(P^{*}\left(k_{0}\right)-I\right)$ the solutions $\nu$ of the equations systems (27) and (28) are analogous (see Theorem 15 and Theorem 19). As in Section 7.1, we initially give a affine transformation of $\gamma$ :

$$
\begin{gathered}
\gamma(s)=\sum_{i=1}^{n} \sum_{w_{i}=0}^{s_{i}}\left(s_{i}-w_{i}\right) q_{0}\left(w_{i}\right)=\sum_{i=1}^{n} \sum_{w_{i}=0}^{k_{0}}\left(s_{i}-w_{i}\right) q_{0}\left(w_{i}\right) \\
\quad-\sum_{i=\eta}^{n} \sum_{w_{i}=s_{i}+1}^{k_{0}}\left(s_{i}-w_{i}\right) q_{0}\left(w_{i}\right)+\left(\overline{s u}-\sum_{i=\eta}^{n}\left(k_{0}-s_{i}\right)\right) q_{0}\left(k_{0}\right) \\
=\sum_{i=1}^{n} s_{i} \sum_{w_{i}=0}^{k_{0}} q_{0}\left(w_{i}\right)-\sum_{i=1}^{n} \sum_{w_{i}=0}^{k_{0}} w_{i} q_{0}\left(w_{i}\right)+\sum_{i=\eta}^{n} \sum_{w_{i}=s_{i}+1}^{k_{0}-1}\left(w_{i}-s_{i}\right) q_{0}\left(w_{i}\right) \\
\quad+\sum_{i=\eta}^{n}\left(k_{0}-s_{i}\right) q_{0}\left(k_{0}\right)+\overline{s u} q_{0}\left(k_{0}\right)-\sum_{i=\eta}^{n}\left(k_{0}-s_{i}\right) q_{0}\left(k_{0}\right) \\
=s u-\sum_{i=1}^{n} \sum_{w_{i}=0}^{k_{0}} w_{i} q_{0}\left(w_{i}\right)+\sum_{i=\eta}^{n} \sum_{w_{i}=s_{i}+1}^{k_{0}-1}\left(w_{i}-s_{i}\right) q_{0}\left(w_{i}\right)+\bar{u} u q_{0}\left(k_{0}\right) \\
=\sum_{i: s_{i} \leq k_{0}-2} \sum_{w_{i}=s_{i}+1}^{k_{0}-1}\left(w_{i}-s_{i}\right) q_{0}\left(w_{i}\right)+\bar{R}\left(n, s u, k_{0}, q\right), \text { where } \\
\bar{R}\left(n, s u, k_{0}, q\right)=s u-\sum_{i=1}^{n} \sum_{w_{i}=0}^{k_{0}} w_{i} q_{0}\left(w_{i}\right)+\overline{s u} q_{0}\left(k_{0}\right) \text { is independent of s. }
\end{gathered}
$$

We define

$$
\begin{gathered}
\gamma^{\prime}(s):=\sum_{i: s_{i} \leq k_{0}-2} \sum_{w_{i}=s_{i}+1}^{k_{0}-1}\left(w_{i}-s_{i}\right) q_{0}\left(w_{i}\right)=\sum_{i: \overline{s_{i}} \geq 2} \sum_{\bar{w}_{i}=1}^{\overline{s_{i}} \text { or }\left(\overline{s_{i}}-1\right)}\left(\bar{s}_{i}-\bar{w}_{i}\right) q_{0}\left(k_{0}-\bar{w}_{i}\right) \\
\text { where } \overline{s_{i}}:=k_{0}-s_{i}
\end{gathered}
$$

and in particular, $\gamma^{\prime}\left(\left(k_{0}, \cdots, k_{0}, k_{0}-1, \cdots, k_{0}-1\right)^{T}\right):=0$.

Using $\gamma^{\prime}(s)$ from (29) in the equation system (28), $g^{\prime}=0$ follows from the last equation (the equation with $\gamma^{\prime}\left(s^{r}=\left(k_{0}, \cdots, k_{0}, k_{0}-1, \cdots, k_{0}-1\right)^{T}\right)$ on the right side) where Corollary 11 is kept in mind. If we fix $\nu\left(s^{r}\right)=0$, the 
following system remains

$$
\begin{gathered}
\sum_{l=1}^{r-1} \lim _{k_{0} \rightarrow \infty} \frac{1}{c\left(k_{0}\right)}\left(p^{*}\left(s^{l} \mid s^{f}\right)-\delta\left(s^{l}, s^{f}\right)\right) \nu\left(s^{l}\right)=-\lim _{k_{0} \rightarrow \infty} \frac{1}{c\left(k_{0}\right)} \gamma^{\prime}\left(s^{f}\right), \\
f=1, \cdots, r-1 .
\end{gathered}
$$

Theorem 19. Let $S_{n ; s u=n k_{0}-s \bar{u} ; k_{0}}, k_{0}=(\overline{s u}), s \bar{s}+1, \overline{s u}+2, \cdots(\overline{s u}<n)$ be sets of non-truncated heavy partitions, each of these sets with $r$ partitions, and with the partial dominance ordering. Furthermore, let $B_{n ; k_{0}}$ be the corresponding sets of requirements, where for any $k_{0}$, the requirements $w_{i},(i=1, \cdots, n)$ are independent and identically distributed and where (10) is assumed. In addition, let corresponding given probability functions $q^{k_{0}}$ fulfill (20) and (21) for certain $c\left(k_{0}\right)$. Finally, let $P^{*}\left(k_{0}\right)$ be the the corresponding PRMs. Then, $\nu\left(\left(k_{0}, \cdots, k_{0}, k_{0}-1, \cdots, k_{0}-1\right)^{T}\right):=0$,

$$
\begin{array}{r}
\nu(s)=\sum_{i: s_{i} \leq k_{0}-2}\left(\frac{q_{0}^{0}\left(k_{0}-1\right)}{q_{0}^{0}\left(k_{0}\right)+q_{0}^{0}\left(k_{0}-1\right)}+\frac{q_{0}^{0}\left(k_{0}-1\right)+q_{0}^{0}\left(k_{0}-2\right)}{q_{0}^{0}\left(k_{0}\right)+q_{0}^{0}\left(k_{0}-1\right)+q_{0}^{0}\left(k_{0}-2\right)}+\cdots\right. \\
\left.\quad+\frac{q_{0}^{0}\left(k_{0}-1\right)+q_{0}^{0}\left(k_{0}-2\right)+\cdots+q_{0}^{0}\left(s_{i}+1\right)}{q_{0}^{0}\left(k_{0}\right)+q_{0}^{0}\left(k_{0}-1\right)+\cdots+q_{0}^{0}\left(s_{i}+1\right)}\right) \\
\left(\text { where } q_{0}^{0}\left(w_{i}\right):=\lim _{k_{0} \rightarrow \infty} \frac{q_{0}^{k_{0}\left(w_{i}\right)}}{c\left(k_{0}\right)}\right) \\
=\sum_{i: s_{i} \leq k_{0}-2}\left(k_{0}-s_{i}-q_{0}^{0}\left(k_{0}\right)\left(\frac{1}{Q_{0}^{0}\left(k_{0}\right)}+\frac{1}{Q_{0}^{0}\left(k_{0}-1\right)}+\cdots+\frac{1}{Q_{0}^{0}\left(s_{i}+1\right)}\right)\right) \\
\text { where } Q_{0}^{0}(w)=q_{0}^{0}\left(k_{0}\right)+q_{0}^{0}\left(k_{0}-1\right)+\cdots+q_{0}^{0}(w)
\end{array}
$$

are solutions of the equation systems (28) with $\gamma^{\prime}$ from (29).

Proof. We use the following notations in relation to partitions:

$$
\begin{aligned}
& s\left(k_{0}\right)=\left(k_{0}, \cdots, k_{0}, k_{0}-1, \cdots, k_{0}-1, k_{0}-\bar{s}_{\eta}, \cdots, k_{0}-\bar{s}_{n}\right) \\
& \text { with } 2 \leq \bar{s}_{\eta} \leq \bar{s}_{\eta+1} \leq \cdots \leq \bar{s}_{n} \leq k_{0}-1
\end{aligned}
$$

(see Definition 9(a) for $\eta$ ) $\bar{s}_{h_{j}}=\bar{s}_{h_{j+1}}=\cdots=\bar{s}_{h_{j+1}-1}$,

$j=1,2, \cdots, \alpha$ where $\eta=h_{1}<h_{2}<\cdots<h_{\alpha} \leq n:=h_{\alpha+1}-1$.

Furthermore, we note

$$
\begin{aligned}
& \nu\left(s_{i}\right):= \frac{q_{0}^{0}\left(k_{0}-1\right)}{q_{0}^{0}\left(k_{0}\right)+q_{0}^{0}\left(k_{0}-1\right)}+\cdots+\frac{q_{0}^{0}\left(k_{0}-1\right)+q_{0}^{0}\left(k_{0}-2\right)+\cdots+q_{0}^{0}\left(s_{i}+1\right)}{q_{0}^{0}\left(k_{0}\right)+q_{0}^{0}\left(k_{0}-1\right)+\cdots+q_{0}^{0}\left(s_{i}+1\right)} \\
& \quad \text { for } s_{i} \leq k_{0}-2, \nu\left(k_{0}-1\right):=0, \nu\left(k_{0}\right):=0, \\
&\left.\gamma^{\prime}\left(s_{i}\right):=\sum_{w_{i}=s_{i}+1}^{k_{0}-1}\left(w_{i}-s_{i}\right) q_{0}\left(w_{i}\right) \text { for } s_{i} \leq k_{0}-2 \text { (this means } i \geq \eta\right)(\operatorname{see}(29)) .
\end{aligned}
$$

Since $\nu\left(\left(k_{0}, \cdots, k_{0}, k_{0}-1, \cdots, k_{0}-1\right)^{T}\right)=0$, we have to prove the identity (see $(30))$

$$
\sum_{l=1}^{r-1} \lim _{k_{0} \rightarrow \infty} \frac{1}{c\left(k_{0}\right)}\left[p^{*}\left(s^{l}\left(k_{0}\right) \mid s\left(k_{0}\right)\right)-\delta\left(s^{l}\left(k_{0}\right), s\left(k_{0}\right)\right)\right] \nu\left(s^{l}\left(k_{0}\right)\right)
$$




$$
=-\lim _{k_{0} \rightarrow \infty} \frac{1}{c\left(k_{0}\right)} \gamma^{\prime}\left(s\left(k_{0}\right)\right)
$$

(where $\gamma^{\prime}(\cdot)$ is from $(29)$ and $\nu(\cdot)$ from this theorem) for any partitions $s\left(k_{0}\right) \neq\left(k_{0}, \cdots, k_{0}, k_{0}-1, \cdots, k_{0}-1\right)^{T}$. Here,

$\lim _{k_{0} \rightarrow \infty} \frac{1}{c\left(k_{0}\right)} p^{*}\left(s^{l}\left(k_{0}\right) \mid s\left(k_{0}\right)\right)=0$ if $s^{l}\left(k_{0}\right)$ is not a restricted monotone successor of $s\left(k_{0}\right)$ (see Theorem 10). Hence, we can replace the sum over $l=1, \cdots, r-1$ in $\left({ }^{*} 2\right)$ with a sum over the restricted monotone successors of $s\left(k_{0}\right) . s^{l}\left(k_{0}\right)$ are restricted monotone successors of $s\left(k_{0}\right)$ if $s^{l}\left(k_{0}\right)_{i} \in\left\{k_{0}, k_{0}-1\right\}$ for $i=1, \cdots, \eta-1, s^{l}\left(k_{0}\right)_{i_{0}}=k_{0}-\bar{s}_{i_{0}} \geq k_{0}-\bar{s}_{i_{0}}=s\left(k_{0}\right)_{i_{0}}$ for one $i_{0} \in$ $\{\eta, \cdots, n\}$ and $s^{l}\left(k_{0}\right)_{i}=s\left(k_{0}\right)_{i} \quad$ for $i \in\{\eta, \cdots, n\} \backslash\left\{i_{0}\right\}$ (see Definition $9(\mathrm{~b}))$. This implies the summation $\sum_{i=1}^{\alpha} \sum_{\bar{s}^{\prime} h_{i}=1}^{\bar{s}_{h_{i}}} \ldots$ as realization of the summation over the restricted monotone successors of $s\left(k_{0}\right)$. Here the summand $\lim _{k_{0} \rightarrow \infty} \frac{1}{c\left(k_{0}\right)}\left(p^{*}\left(s\left(k_{0}\right) \mid s\left(k_{0}\right)\right)-1\right) \nu\left(s\left(k_{0}\right)\right)=$

$-\sum_{i=\eta}^{n}\left(q_{0}^{0}\left(k_{0}-\bar{s}_{i}+1\right)+\cdots+q_{0}^{0}\left(k_{0}\right)\right) \nu\left(s\left(k_{0}\right)\right) \quad$ (see Theorem 10) is separately noted down. The summations $\sum_{i=1}^{\alpha} \sum_{\bar{s}^{\prime} h_{i}=1}^{\bar{s}_{h_{i}}-\mathbf{1}} \cdots$ then remain.

With this method, we can then give an equation equivalent to equation $\left(*^{2}\right)$ (where, additionally, the formula from Theorem 10 is used for the remaining limits):

$$
\begin{aligned}
& -\sum_{i=\eta}^{n}\left(q_{0}^{0}\left(k_{0}-\bar{s}_{i}+1\right)+\cdots+q_{0}^{0}\left(k_{0}\right)\right) \nu\left(s\left(k_{0}\right)\right) \\
& +\sum_{i=1}^{\alpha}\left(h_{i+1}-h_{i}\right)\left[\sum_{\bar{s}^{\prime} h_{i}=1}^{s_{h_{i}}-1} q_{0}^{0}\left(k_{0}-\bar{s}^{\prime} h_{i}\right)\left(\nu\left(s\left(k_{0}\right)\right)-\nu\left(k_{0}-\bar{s}_{h_{i}}\right)+\nu\left(k_{0}-\bar{s}^{\prime} h_{i}\right)\right)\right. \\
& =-\lim _{k_{0} \rightarrow \infty} \frac{1}{c\left(k_{0}\right)} \gamma^{\prime}\left(s\left(k_{0}\right)\right),
\end{aligned}
$$

where according to the formulas for $\nu(s)$ (from this theorem) and $\nu\left(s_{i}\right)$ (from the above proof): $\nu\left(s\left(k_{0}\right)\right)-\nu\left(k_{0}-\bar{s}_{h_{i}}\right)+\nu\left(k_{0}-\bar{s}^{\prime} h_{i}\right)=\nu\left(s^{l}\left(k_{0}\right)\right)$ for $s^{l}\left(k_{0}\right)$ with $s^{l}\left(k_{0}\right)_{h_{i}}=k_{0}-\bar{s}^{\prime} h_{i}$ and $s^{l}\left(k_{0}\right)_{j}=s\left(k_{0}\right)$ for $j \neq h_{i}$ and thus, in particular, $\nu\left(s\left(k_{0}\right)\right)-\nu\left(k_{0}-\bar{s}_{h_{i}}\right)=\nu\left(s^{l}\left(k_{0}\right)\right)$ if $\bar{s}^{\prime} h_{i}=k_{0}-1$. A simple reorganization of the sums yields

$$
\left\{-\sum_{i=\eta}^{n}\left(q_{0}^{0}\left(k_{0}-\bar{s}_{i}+1\right)+\cdots+q_{0}^{0}\left(k_{0}\right)\right)\right.
$$




$$
\begin{aligned}
& \left.+\sum_{i=1}^{\alpha}\left(h_{i+1}-h_{i}\right) \sum_{\bar{s}^{\prime} h_{i}=\mathbf{0}}^{\bar{s}_{h_{i}}-1} q_{0}^{0}\left(k_{0}-\bar{s}^{\prime} h_{i}\right)\right\} \nu\left(s\left(k_{0}\right)\right) \\
& +\sum_{i=1}^{\alpha}\left(h_{i+1}-h_{i}\right) \sum_{\bar{s}^{\prime} h_{h_{i}}=1}^{\bar{s}_{h_{i}}-1} q_{0}^{0}\left(k_{0}-\bar{s}^{\prime} h_{i}\right)\left(-\nu\left(k_{0}-\bar{s}_{h_{i}}\right)+\nu\left(k_{0}-\bar{s}^{\prime} h_{i}\right)\right) \\
& +\sum_{i=1}^{\alpha}\left(h_{i+1}-h_{i}\right) q_{0}^{0}\left(k_{0}\right)\left(-\nu\left(k_{0}-\bar{s}_{h_{i}}\right)\right) \\
& =-\lim _{k_{0} \rightarrow \infty} \frac{1}{c\left(k_{0}\right)} \gamma^{\prime}\left(s\left(k_{0}\right)\right) .
\end{aligned}
$$

Since $\sum_{i=1}^{\alpha}\left(h_{i+1}-h_{i}\right) \cdots$ can be replaced with $\sum_{i=\eta}^{n} \cdots, \quad$ the term in the parentheses $\{\cdots\}$ yields 0 and the following equivalent equation remains:

$$
\begin{aligned}
& \sum_{i=\eta}^{n} \sum_{\bar{s}^{\prime}{ }_{h_{i}}=1}^{\bar{s}_{h_{i}}-1} q_{0}^{0}\left(k_{0}-\bar{s}^{\prime}{ }_{h_{i}}\right)\left(-\nu\left(k_{0}-\bar{s}_{h_{i}}\right)+\nu\left(k_{0}-\bar{s}^{\prime} h_{i}\right)\right)+\sum_{i=\eta}^{n} q_{0}^{0}\left(k_{0}\right)\left(-\nu\left(k_{0}-\bar{s}_{h_{i}}\right)\right) \\
& \quad=-\lim _{k_{0} \rightarrow \infty} \frac{1}{c\left(k_{0}\right)} \gamma^{\prime}\left(s\left(k_{0}\right)\right) . \text { Finally, } \\
& \quad-\sum_{i=\eta}^{n}\left\{\sum_{\bar{s}^{\prime}{ }_{h_{i}}=\mathbf{0}}^{\bar{s}_{h_{i}}-1} q_{0}^{0}\left(k_{0}-\bar{s}^{\prime} h_{i}\right) \nu\left(k_{0}-\bar{s}_{h_{i}}\right)-\sum_{\bar{s}^{\prime} h_{i}=1}^{\bar{s}_{h_{i}}-1} q_{0}^{0}\left(k_{0}-\bar{s}^{\prime} h_{i}\right) \nu\left(k_{0}-\bar{s}^{\prime} h_{i}\right)\right\} \\
& =-\sum_{i=\eta}^{n}\left\{\lim _{k_{0} \rightarrow \infty} \frac{1}{c\left(k_{0}\right)} \gamma^{\prime}\left(s\left(k_{0}\right)_{i}\right)\right\} .
\end{aligned}
$$

Here $\{\cdots\}=\{\cdots\}$ is valid. This relationship is analogous to $(* 4)$ from the proof of Theorem 15 and could be proven in an analogous way. Thus, the assertion of the Theorem is confirmed since we have only used equivalent transformations of the equations.

Similar to Corollary 16, in relation to sets of sparse partitions, Theorem 19 implies here:

Corollary 20. Let the same assumptions as in Theorem 19 be valid. Furthermore, let $s^{f}$ be a (direct) predecessor of $s^{l}$, where $\left\{s^{f}, s^{l}\right\} \subseteq S_{n ; s u ; k_{0}}$ for any $k_{0} \underset{(=)}{>} \overline{s u}$ and $s^{f} \neq s^{l}$. Then, $\nu\left(s^{l}\right)<\nu\left(s^{f}\right)$ is valid for solutions of the equation system (28). This means that the solutions of the equation systems (28) are monotone (with respect to the partial order).

Corollary 21. Let the same assumptions as in Theorem 19 be valid. Then, the solutions of the Poisson equations (see (15)) with regard to sets $S_{n ; s u ; k_{0}}$ of non-truncated heavy partitions, are monotone for sufficiently large $n$ and $k_{0}$.

In order to prove this corollary we use considerations analogous to those for 
the case of sets of sparse partitions, refer to Corollary 17 . We must additionally note that $\gamma^{\prime}$ (see (29)), the limits of PRMs $P^{*}\left(k_{0}\right)$, as $k_{0}$ tends to infinity, and the solutions $\nu$ of the corresponding equation systems depend on the principal parts of the partitions, however not on $n$.

Corollary 22. Let the same assumptions as in Theorem 19 be valid. This

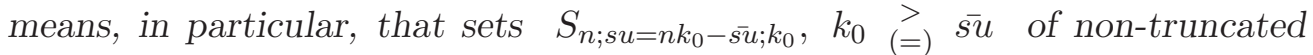
heavy partitions, each of these sets with $r$ partitions, are considered. The corresponding Poisson equations, with exception of perhaps a finite number of them, then have monotone solutions.

\section{Conclusions for the Special SDDP Problem}

\section{Corollary 23. .}

(i) Let reduced SDDP problems, as in Section 3, with

a) m-totally ordered state spaces or

b) state spaces with at most 4 partitions and discrete uniformly distributed requirements in the case of exactly 4 partitions

be given. Then, decisions for feasible states (unordered partitions) with least square sums of their components are optimal for such reduced SDDP problems.

(ii) Let reduced SDDP problems, as in Section 3, with state spaces

$$
\left\{\begin{array}{c}
S_{n ; s u ; k_{0}}, n=(s u,) s u+1, s u+2, \cdots \quad \text { (sets of sparse partitions, } \\
s u \text { and } k_{0} \text { arbitrary but fixed) or } \\
S_{n ; s u=n k_{0}-\bar{u} ; k_{0},}, k_{0}=(\overline{s u} ;) \bar{u} u+1, \overline{s u}+2, \cdots(\overline{s u}<n) \text { (sets of } \\
\text { non-truncated heavy partitions, } \bar{u} u \text { arbitrary but fixed) }
\end{array}\right.
$$

each of these sets with $\left\{\begin{array}{l}r_{1} \\ r_{2}\end{array}\right.$ partitions, be given. Furthermore, let the probability functions of the requirements of the corresponding sets of requirements be as in Theorem 15 or Theorem 19, respectively. Then, decisions for feasible states with least square sums of their components are optimal for such reduced SDDP problems, with exception of perhaps a finite number of them. 


\section{Conclusions}

The SDDP problems is a complex Operations Research problem. Superordinately regarded, SDDP problems are stochastic dynamic programming problems. On the other hand, the problem shows combinatorial aspects. Under the assumption of identical basic costs and independent and identically distributed requirements we showed by means of combinatorial ideas in many cases that decisions for feasible states with least square sums of their parts are optimal solutions. It was not simple to prove that optimally conjectured decisions are in fact optimal if the "conditions of dominance" was infringed on. Since no formulas are known for the corresponding matrices of transition probabilities (PRMs) we used limits of PRMs. For this purpose, we classified sets of partitions and considered sets of "sparse partitions" and sets of "non-truncated heavy partitions". Investigations of special cases and of inherent characteristic structures of SDDP problems are also important as a basis for heuristics.

\section{References}

[1] A. Borodin, R. El-Yaniv, Online Computation and Competitive Analysis, University Press, Cambrigde 1998.

[2] R. Hildenbrandt, A special stochastic decision problem, Optimization, 28 (1993), 95-110.

[3] R. Hildenbrandt, Eine Verbandsstruktur für Partitionen ganzer Zahlen, http ://www:db?thueringen.de/servlets/DocumentServlet?id=5664

[4] R. Hildenbrandt, Methoden aus ganzzahliger Optimierung und Verbandstheorie zur Behandlung eines stochastischen dynamischen Transportproblems, Habilitation, TU Ilmenau (1995).

[5] R. Hildenbrandt, Partitions-requirements-matrices, Symposium on Operations Research, Duisburg 9/2001 (Proceedings, Springer, Berlin, Heidelberg (2002), 303-311.

[6] R. Hildenbrandt, Stochastic dynamic programming with random disturbances, Discussiones Mathematicae Probability and Statistics, 23 (2003), 5-4, doi: 10.7151/dmps

[7] A.W. Marshall, I. Olkin, Inequalities: Theory of Majorization and its Applications, Mathematics in Science and Engineering, Vol. 143. Academic Press, New York (1979). 
[8] P.H. Müller, V. Nollau, Steuerung Stochastischer Prozesse, AkademieVerlag, Berlin (1984).

[9] M. Puterman, Markov Decision Processes, Wiley (1994).

[10] W. Szwarc, The Dynamic transportation problem, Mathematica, 81 (1971), 335-345. 
